\title{
A flexible potential-flow model based high resolution spatiotemporal energy demand forecasting framework
}

Peng, Jieyang; Kimmig, Andreas; Niu, Zhibin; Wang, Jiahai; Liu, Xiufeng; Ovtcharova, Jivka

Published in:

Applied Energy

Link to article, DOI:

10.1016/j.apenergy.2021.117321

Publication date:

2021

Document Version

Early version, also known as pre-print

Link back to DTU Orbit

Citation (APA):

Peng, J., Kimmig, A., Niu, Z., Wang, J., Liu, X., \& Ovtcharova, J. (2021). A flexible potential-flow model based high resolution spatiotemporal energy demand forecasting framework. Applied Energy, 299, [117321]. https://doi.org/10.1016/.apenergy.2021.117321

\section{General rights}

Copyright and moral rights for the publications made accessible in the public portal are retained by the authors and/or other copyright owners and it is a condition of accessing publications that users recognise and abide by the legal requirements associated with these rights.

- Users may download and print one copy of any publication from the public portal for the purpose of private study or research.

- You may not further distribute the material or use it for any profit-making activity or commercial gain

- You may freely distribute the URL identifying the publication in the public portal 


\title{
A flexible potential-flow model based high resolution spatiotemporal energy demand forecasting framework
}

\author{
Peng Jieyang ${ }^{\mathrm{a}, \mathrm{b}}$, Andreas Kimmig ${ }^{\mathrm{b}}$, Niu Zhibin ${ }^{\mathrm{c}, \mathrm{d}, *}$, Wang Jiahai ${ }^{\mathrm{a}, *}$, Xiufeng Liu ${ }^{\mathrm{c}}$, Jivka Ovtcharova ${ }^{\mathrm{b}}$ \\ ${ }^{a}$ Advanced manufacturing technology center, Tongji university, 200092 Shanghai, PR China \\ ${ }^{b}$ Karlsruhe Institute of Technology, 76133 Karlsruhe, Germany \\ ${ }^{c}$ Department of Technology, Management and Economics, Technical University of Denmark, 2800 Kgs. Lyngby, Denmark \\ ${ }^{d}$ College of Intelligence and Computing,Tianjin University, 300354, China
}

\begin{abstract}
Aiming to become more sustainable by transitioning to renewablec energy resources, the energy efficiency problems need to be addressed. Understanding urban demand profiles is an important determinant for energy dispatch and the optimization of the electric energy supply. For the design of the energy supply system, an important consideration is, to express the characteristics of urban household energy demand as a function of space and time. However, the focus of most research activities is only on the modeling of time series data. High-resolution forecasting models for the spatial-temporal distribution of energy were rarely reported in current literature. In this paper, we propose a spatiotemporal forecasting model based on potential-flow for urban energy demand forecasting. Compared with previous studies, potential-flow can describe energy migration in space with a high resolution. Based on the orientation of vectors, our model can predict the direction and intensity of spatial migrations in energy demand and identify energy transfer events. Extensive experiments on real-world data sets verify that our approach can achieve a better prediction accuracy compared with traditional methods. In further empirical studies, we find that the temporal electricity demand flow shows locally concentrating behaviour for different regions of the city. In addition to temporal factors such as seasons, peaks and valleys, such clustering behavior also depend on local populations and major industries (financial, commercial, residential, etc.). Finally, we use entropy to quantitatively describe the intensity of this clustering phenomenon and explore its relationship with meteorological factors. Our research demonstrates a unified visual prediction approach to support exploratory demand analysis. We anticipate that the process will be expanded to support more forms of energy in the future.
\end{abstract}

Keywords: Urban energy, Spatio-temporal demand forecasting, Visual analysis, Spatial mapping, Pattern recognition

\section{Introduction}

Solving urban energy prediction problems is essential for operating, monitoring, and optimizing diverse energy systems, from the supply side to the demand side [1]. Knowledge of future energy consumption for different time

\footnotetext{
*corresponding author: Niu Zhibin, Wang jiahai should also be considered as Co-coresspondingauthors

Email address: zhini@dtu.dk (Niu Zhibin)
} 
periods ranging from minutes to several days is one of the key requirements in supplying energy to the end-consumer in a secure and economical way [2]. On the other hand, this knowledge enables the optimization of the daily operation of urban energy systems, including economic scheduling, unit commitment, scheduled maintenance, load dispatch and security assessment [3]. Using these advanced demand forecast approaches in these highly capital- and technologyintensive energy systems [4], therefore, would lead to a significant improvement in both reducing operating cost and power supply reliability [5].

Functionally, energy forecasting can be divided into three types: time-series forecasting, spatial energy analysis, and spatio-temporal forecasting. Spatial analysis is premised on the assumption that energy consumption is usually cyclical (weekly, yearly, etc.), and the study of the spatial distribution of energy consumption in a certain region can provide insight into future energy consumption. Spatial energy analysis usually analyzes regional energy consumption differences at the city scale to support future urban planning. Time-series forecasting refers to the prediction of electricity consumption in a certain region for a period of time in the future, which is usually used to provide a reference for setting future electricity prices and formulating corresponding energy policies to control energy consumption within a reasonable range on a macro scale. Most current urban energy demand forecasting methods are usually limited to urban-scale time series forecasting, although they may include comparisons between different cities or regions within cities. Whereas time series forecasting may suffice for the overall planning of urban energy resources, only highly spatial and temporal resolution enables capturing the dynamic spatial nature. As the number, volume and resolution of spatio-temporal data increase rapidly, traditional data mining methods, especially statistics-based methods for dealing with such data are becoming overwhelmed [6]. The difficulties of constructing a dynamic spatio-temporal forecasting model of energy demand are listed as follows:

- Insufficient understanding and exploitation of the entanglement of spatial and temporal energy demand information, as most current data-driven forecasting approaches are based solely on sequence-to-sequence models, which are only suitable for time-series data [7]

- Lack of generalization capability and flexibility in various tasks regarding variable resolution and spatiotemporal demand analysis, as most current spatio-temporal energy demand simulation approaches focus on fixed-resolution tasks (e.g., annual or national scale). In addition, most current methods are also unable to analyze the spatio-temporal energy consumption patterns for different user groups.

- Lack of intuition and interpretability, as previous work in this field mainly uses ideal socio-economic models (requiring in-depth knowledge of economics and energy) or data-driven models (generally producing numerical results, requiring machine learning and programming skills), thus with the expected results it can be difficult to understand complex realistic energy demand shifts.

Realistically representing spatio-temporal variations in demand is therefore challenging, and requires data analysis and combination techniques of data [8]. We address this knowledge gap by providing a novel data-driven spatiotemporal forecasting model of urban energy demand. The novelty of this paper lies in the following: 


\begin{tabular}{|llll|}
\hline \multicolumn{2}{l}{ Nomenclature } & LT & Daily low temperature \\
LSTM & Long short-term memory & HT & Daily high temperature \\
DL & Deep learning & DHT & Daily high dew temperature \\
ANN & Artificial neural network & LH & Daily low humidit \\
SVR & Support vector regression & HH & Daily high humidity \\
RFR & Random forest regression & LW & Daily low wind speed \\
ABR & Ada boost regression & HW & Daily high wind speed \\
DT & Decision tree & LA & Daily low air pressure \\
LR & Linear Regression & HA & Daily high air pressure \\
R2 & R squared & AP & Air pollution index \\
MAE & Mean absolute error & WT & Weather type \\
MSLE & Mean Square Logarithmic error & WD & Wind direction \\
RMSE & Root mean squared error & HI & Holiday index \\
GBR & Gradient Boosting Regressor & ANN & Artificial Neural Network \\
\hline
\end{tabular}

- We propose a novel method ("energy demand flow") for modeling the spatio-temporal variation of energy demand based on the potential-flow theory in fluid dynamics.

- We propose a variable-resolution spatio-temporal energy prediction model based on clustering and long shortterm memory neural network, providing unprecedented flexibility for the diverse resolution analysis tasks.

- We develop an intuitive and user-friendly visualization analysis interface and conduct empirical studies, based on the real electricity consumption data of Shanghai for a time period of three years.

- We propose the concept of entropy of energy demand to quantitatively describe the intensity of the clustering phenomenon when energy demand migrates.

This paper constructs a spatio-temporal forecasting model of urban energy resources based on potential flow (also defined as energy demand flow). Taking Shanghai as an empirical case, we provide novel insights into the energy demand distribution in different time or space gradients and the spatio-temporal shift of energy in different consumption patterns. Insights from our work can be used to optimize existing energy supply mechanisms and to support the integration of renewable resources. The remainder of this paper is organized as follows. Section 2 reviews the current approaches in the field of energy demand prediction and then summarizes the difficulties in spatio-temporal energy demand forecasting, as well as the innovations of our work. In Section 3, the case data and the implementation process applied in this paper are presented. Section 4 gives an overview of the prediction results of this method and compares it with other traditional methods to verify the effectiveness of this method. Then, taking Shanghai's three-year electricity consumption data as an example, we developed a spatio-temporal energy forecasting system and conducted an empirical study on the method proposed in this paper, which is presented in Chapter 5. Finally, Section 6 summarizes the main contributions of our work and proposes future research directions. 


\section{Literature review}

This section first discusses the state of the art in urban energy forecasting from the perspective of the demand profiles currently used. Then, the difficulties of spatio-temporal energy prediction and the main innovation points of our work are summarized. Two types of approaches for energy demand forecasting can be identified: top-down and bottom-up. Distinguishing between bottom-up and top-down approaches is common for classifying energy demand models: whereas top-down approaches are rather coarse and not concerned with individual end uses, bottom-up approaches identify homogeneous activities or end uses for more precise demand simulation.

Time series forecasting (top-down). Motivated by the need for energy demand forecasts, first, the top-down approach is applied to the energy demand prediction problem. The top-down approach employs macroeconomic and spatially or temporally aggregated data. Such approaches produce a coarse definition of demand profiles and the structure of energy consumers. Uris et al. first applied the Econometric Model to energy forecasting problems in 1978 [9]. In the same year, Halvorsen discussed residential, commercial and industrial demand for electricity in power generation and dynamic energy demand in manufacturing based on the top-down approach [10]. Liu et al. used the econometric model to predict Singapore's future electricity consumption [11]. Chern et al. proposed an econometric model developed for the US Nuclear Regulatory Commission to conduct an independent assessment of electricity demand forecasts [12]. In general, top-down approaches are not concerned with individual end uses, but rather focus on analyzing the relationship between output, i.e., energy demand, and related factors.

Time series forecasting (bottom-up). The bottom-up approach for forecasting relies on a large amount of historical data to train a model. This approach is also known as data-driven model [13, 14]. However, it requires detailed energy consumption information [16] and the spatial distribution [17, 18, 15] of energy consumers, which are often not available. For the past few years, advanced measurement and communication technologies, such as smart grids, have particularly played a key role in dealing with different energy demand projection challenges faced during the development of cities. These technologies also provide a wealth of real, analyzable data for data-driven approaches in the energy field. Auto-regressive modeling (AR) is one of the most classical forecasting techniques, which is based on statistical analysis of time-series data. It only requires that statistical properties of the time-series should be timeinvariant, or, in other words, that the energy consumption at a specific time should be similar to properties of the recent past [19]. Newsham et al. developed an AR model to forecast occupancy-related electricity load of different research buildings in Canada [20]. Kyungtae et al. implemented four different AR-based models to separately predict an hour-ahead building cooling and heating loads [21]. Dagnely et al. developed an innovative AR model to forecast the electricity load demand of an office in Brussels for the next 72-h [22].

Another type of bottom-up energy forecasting models are statistical regression models, which have been widely implemented for both pre-occupancy and post-occupancy forecasting studies such as energy retrofit impact assessment [19]. For instance, Cyprus used multiple regression analyses to determine the relationship between energy consumption, the number of customers, the price of electricity and the number of tourists [25]. Amber et al. im- 
plemented a regression model to predict the daily electricity consumption in London [23]. Pulido et al. developed a regression based forecasting model for an office building in Chile using a government database [24]. Traditional regression models are also used for short term [26, 27, 28] and long term load forecasting [29]. However, traditional regression models are generally for linear data, which is difficult in dealing with nonlinear data or regression missions with complex correlations between features. For nonlinear problems, random forest(RF), support Vector Regression(SVR) [30], XGBoost [31] etc. are widely used in energy prediction and evaluation projects, such as predictive control for building energy optimization [32] and solar photo-voltaic technical potential estimation [33].

In recent years, deep learning models have played an increasingly prominent role in the field of energy prediction. Artificial Neural Networks (ANNs) are highly flexible and adaptable models, which can solve most forecasting problems, including non-linear patterns. They have been widely used for energy load forecasting. Aydinalp et al. used ANN to model the energy consumption of appliances, lighting, and space-cooling in the Canadian residential sector [34]. Sozen determined sectoral energy consumption and greenhouse gas prediction in Turkey using an ANN model [35]. Geem estimated the energy demand for South Korea by ANN model, taking into account gross domestic product, population, import and export index [36]. Gonzalez analysed the monthly electric demand for Spain using a hybrid NN model [37]. Hsiao et al. forecast the electricity consumption of Taiwan using ANN. In their work, four economic factors, namely the national income, population, gross of domestic production, and consumer price index are used to study the influence on the electricity consumption [38]. In recent years, various variants of neural networks (usually combined with other models) have been widely used to improve energy prediction accuracy. Jallal incorporated the adaptive neuro-fuzzy inference model with the firefly algorithm denominated as the gender-difference firefly algorithm [39]. Theocharides combines a neural network with statistical post-processing for hourly averaged day-ahead photo-voltaic power production forecasting with improved accuracy [40]. Bhattacharyya and Swan respectively conducted a literature review on data-driven models in the field of energy forecasting [41, 42]. Data-driven approaches are the preferable option for urban scale energy system modeling, as they can identify homogeneous activities or end uses for demand simulation and result in detailed descriptions of demand variations.

Spatial-temporal forecasting model. With the urban scale becoming more important in energy system modeling for the green energy transition, both spatial and temporal demand fluctuations become relevant [43]. To underscore the importance of both dimensions, high resolution spatio-temporal demand modelling [45] is crucial for coupling energy demand models with energy supply models operating at similar tempo-spatial scales for energy system optimisation. Specifically, temporal high resolution is critical for modeling demand side management and storage and thus to simulate future changes in peak demand [44], while spatial detail is necessary for understanding regional energy needs and long-term capacity planning as well as the spatial allocation of energy demand and supply [46]. However, only a limited number of studies are available, including both spatial and temporal effects of energy demand integration. Heuberger proposed a spatio-temporal granular model for electricity systems to investigate the impact of electric vehicle deployment [47]. Yang applied an ensemble structure-based neural network model to analyze the city-level carbon emissions, and consequently promote a better understanding of the spatio-temporal characteristics 
of carbon emissions [48]. Most research on spatio-temporal energy demand is to summarize the existing knowledge of spatio-temporal energy consumption by analyzing historical data through simulation [46] or modeling [8], which fails to provide accurate prediction results [49].

\section{Methods}

In this section, we first present the overall framework of our model, then we introduce the case data of this paper, and finally we provide detailed information on the implementation and theory of each step.

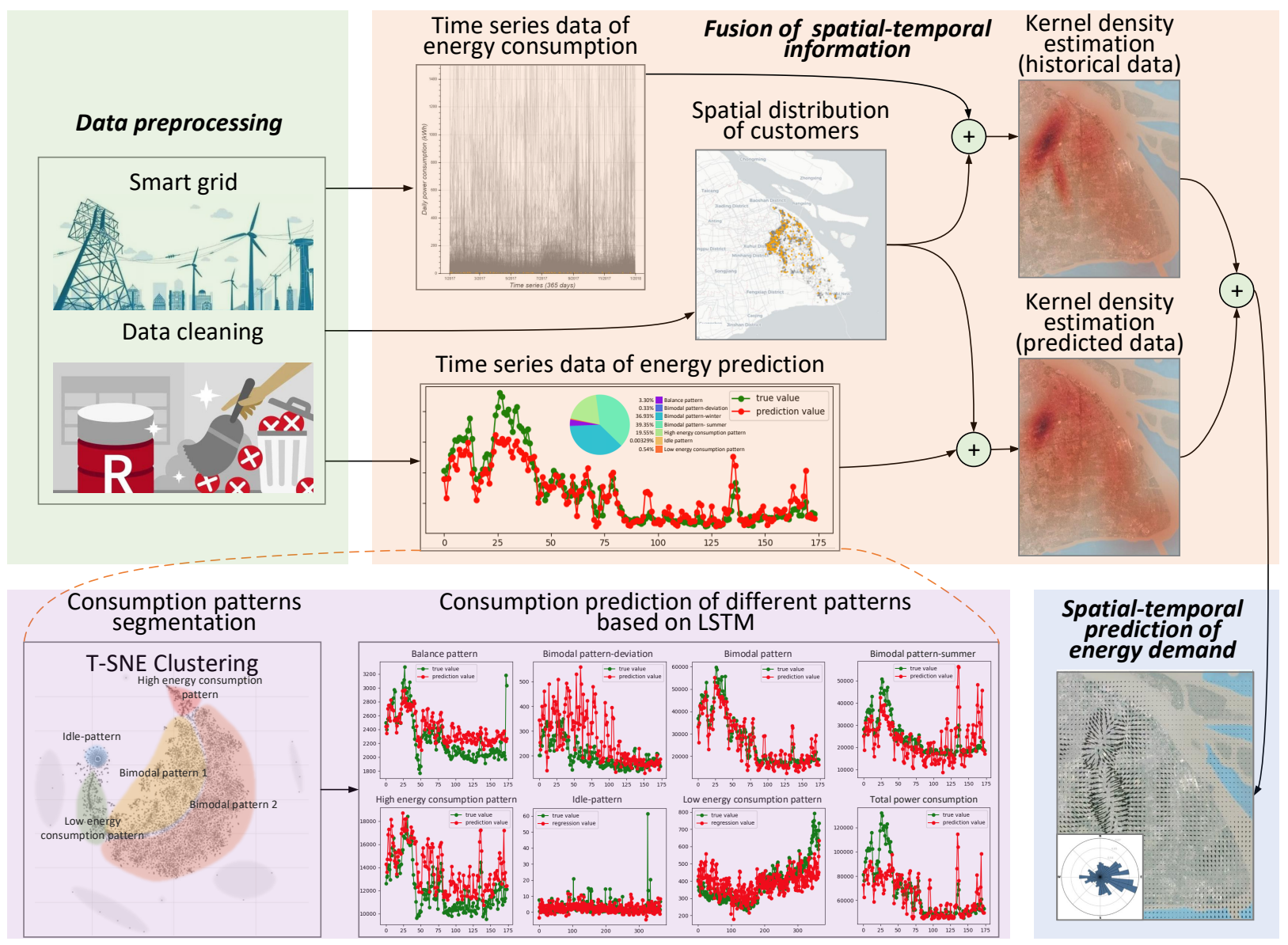

Figure 1: Overview of the proposed framework. The modules in the diagram involve two attributes, namely, time attributes (historical, future) and content attributes (temporal, spatial). The time and content attributes involved in each module are marked in the upper-left and upper-right corner of each module, respectively.

\subsection{Modeling framework}

In this paper, a spatio-temporal integration framework is proposed to predict future energy demand from a spatiotemporal perspective. Figure 1 provides a conceptual overview of the approach. The overall procedure includes three 
successive stages: data preprocessing, fusion of spatio-temporal information, and assessment of spatial and temporal distribution of energy.

Data preprocessing aims to improve the data quality for modeling, which is shown on a green background in figure 1. The cleaned data can be divided into time-series data and spatial data. Time series data, on the one hand, is specifically fused with spatial data and, on the other hand, is used to forecast potential energy demand. Potential energy demand will then also be integrated with spatial data.

The second phase of the framework is the fusion stage of spatio-temporal information (The module with an orange background in figure 11. To combine temporal and spatial information, a kernel density estimation approach is proposed to encode discrete household energy consumption into a continuous representation. The advantage of the kernel density estimation is, that it can efficiently generate a smoother vector field. At this phase, the kernel density estimation method is performed twice to encode historical and future geospatial energy demand, respectively. The historical geospatial energy demand is based on the historical energy consumption data, while the future demand is derived from the energy prediction results from the next module. The future energy demand forecasting process is shown on purple background in figure 1 The main function of this stage is to use a clustering-based LSTM to divide the customer set according to energy consumption patterns, and then predict the future energy consumption for each pattern of customers. The purpose of dividing the customer set into various energy consumption patterns is to raise the signal-to-noise ratio of the aggregated time series, and the prediction accuracy of the aggregated ones will be improved [51]. Specifically, the cleaned energy data will be fed to the t-distributed stochastic neighbor embedding (t-SNE) algorithm for energy consumption pattern recognition [53]. The long short term memory neural network will then be applied to predict the future energy demands of different patterns. Finally, the future energy consumption of all patterns is added up to obtain the total energy demand of the city.

The third phase is spatio-temporal demand shift modeling, as shown in the blue background in figure 1 . On the basis of historical and future geospatial energy demand, a spatio-temporal shift model based on the potential-flow field is proposed to represent the spatio-temporal variation trend of potential energy demand. Finally, an entropy-based evaluation index is constructed to quantify the potential energy demand.

\subsection{Data and preprocessing}

In general, spatio-temporal energy demand in urban areas is assessed using geographic information systems combined with time series data on energy consumption. The above data can be obtained from a smart grid, a power plant or the city's statistics bureau. The spatial and temporal prediction model of energy demand proposed here, can be applied to other energy data such as district heating, natural gas and solar energy. The empirical data in this paper is a multi-grained time serial record of the electricity consumption of households, together with their geospatial information, as shown in figure 2

The table on the left in figure 2 shows the sample rows of the original data. The first and second columns are the consumer's location coordinates, expressed in longitude and latitude. Starting from the third column is the time series 


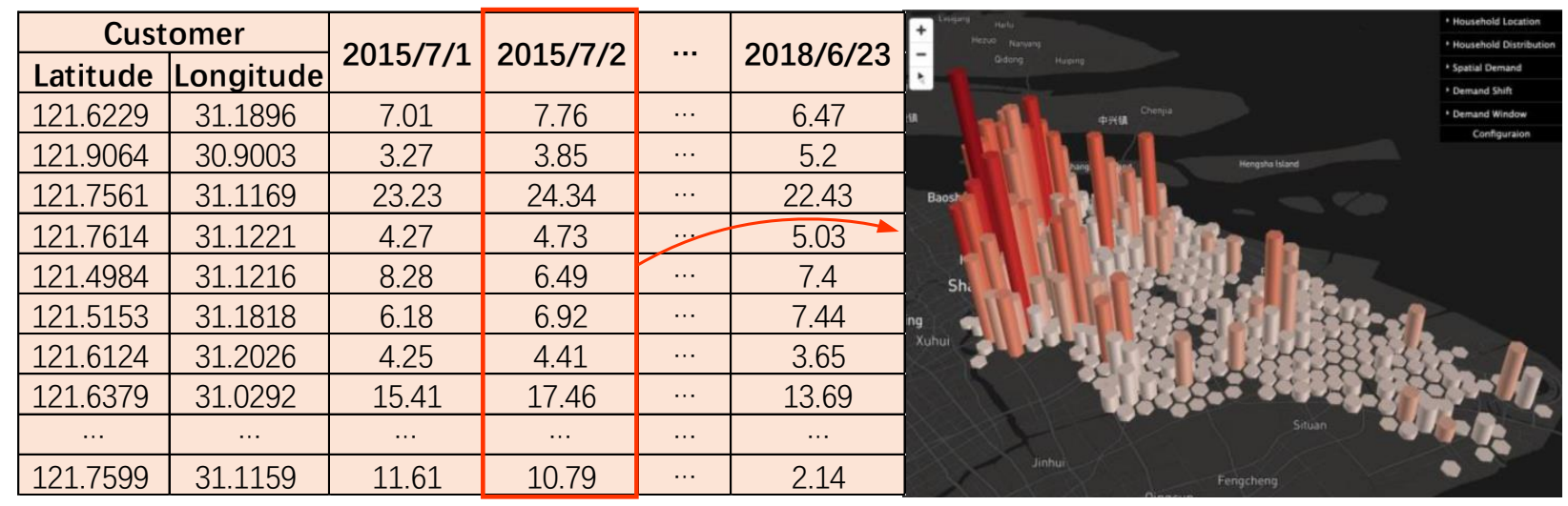

Figure 2: Household electricity consumption data in Shanghai.

data for each consumer's electricity consumption over a period of three years. The three-dimensional histogram on the right reflects the geographical distribution of electricity consumption in Shanghai on a certain day. Therefore, the spatial-temporal variation of energy consumption is a three-dimensional dynamic process.

The missing values in the data set account for approximately $1 \%$ of the total data volume, which may be caused by electricity meter failures or faulty recordings. Considering that the household electricity consumption pattern is unlikely to change drastically within a few days, we use linear interpolation to fill in the empty values. In addition, there are also a few outliers in the data set, which can be reflected by the sudden and abnormal increase in power consumption. In this paper, Tukey's method is adopted to identify outliers, which defines outliers as those values of the data set that fall far from the central point [50]. Then, the mean value of adjacent values is used to replace outliers. Other outlier processing methods include Minkowski error, Multivariate methods, etc. It should be noted that it is necessary to select appropriate outlier processing methods according to the characteristics of different energy data sets.

\subsection{Time series energy prediction based on consumption patterns clustering}

In this paper, we use a clustering-based approach for energy demand forecasting that is similar to Pang [51] but with more flexibility, intuition and interpretability. Instead of dealing with the geographical hierarchy directly, we apply a time series clustering analysis for electricity consumption pattern detection.

\subsubsection{Segmentation of energy consumption patterns}

To some extent, individual household energy consumption (power consumption in this case) usually follows a steady pattern of growth over time. These patterns can be identified by mapping the energy consumption temporal data to the lower dimensional space.

Let $x_{1}, \ldots x_{N}$ be the time series data of urban power consumption, $\mathrm{N}$ is the number of households. The probabilities 
$p_{i j}$ that are proportional to the similarity of objects $x_{i}$ and $x_{j}$ are first computed, as follows.

$$
p_{i j}=\frac{p_{i \mid j}+p_{j \mid i}}{2 N}
$$

Among them, $p_{i \mid j}$ is defined as

$$
p_{i \mid j}=\frac{\exp \left(-\left\|x_{i}-x_{j}\right\|^{2}\right)}{\sum_{k \neq l} \exp \left(-\left\|x_{k}-x_{i}\right\|^{2}\right)}
$$

and note that $p_{i j}=p_{j i}, p_{i i}=0, \sum_{i j} p_{i j}=1$

To learn a low-dimensional map $y_{1}, \ldots y_{N}$ that reflects the similarities $p_{i j}$ as well as possible, a t-distribution is used to measure similarities between low-dimensional points in order to allow dissimilar objects to be modeled far apart in the map [53], define $q_{i j}$ as

$$
q_{i j}=\frac{\left(1+\left\|y_{i}-y_{j}\right\|^{2}\right)^{-1}}{\sum_{k \neq l}\left(1+\left\|y_{k}-y_{l}\right\|^{2}\right)^{-1}}
$$

In order to make the distribution in high and low dimensions as similar as possible, the locations of the points $y_{i}$ in the low-dimensional map are determined by minimizing the Kullback-Leibler divergence of the distribution $P$ from the distribution $Q$, that is:

$$
K L(P \| Q)=\sum_{i} \sum_{j} p_{i j} \log \frac{p_{i j}}{q_{i j}}
$$

The minimization of the Kullback-Leibler divergence with respect to the points $y_{i}$ is performed using gradient descent. The gradient is as follows:

$$
\frac{\delta K L}{\delta y_{i}}=4 \sum_{j}\left(p_{i j}-q_{i j}\right)\left(y_{i}-y_{j}\right)\left(1+\left\|y_{i}-y_{j}\right\|^{2}\right)^{-1}
$$

The clustering results of Shanghai's electricity consumption data are shown in figure 3 . Each point in the cluster map represents a household, and the distance between points represents the difference in power consumption patterns. The horizontal axis of each of the eight subgraphs corresponds to 365 days (1 year), and the vertical axis represents the power consumption on each day. The electricity consumption patterns can be classified into eight categories. The bimodal patterns were found to be the most common, and were subdivided into summer and winter patterns. These two energy consumption patterns can be explained by the use of energy for heat during sun-deprived winter periods and energy for cooling to help tolerate hot summers. High energy consumption and energy saving patterns correspond to two extremes of electricity consumption. There were far fewer households showing either of these extremes than there were showing bimodal patterns. The balance pattern refers to households with a relatively balanced electricity consumption throughout the year, and their energy consumption habits seem to be unaffected by the season. The deviation pattern is similar to the bimodal pattern, with two peak periods of power consumption in a year. Different 


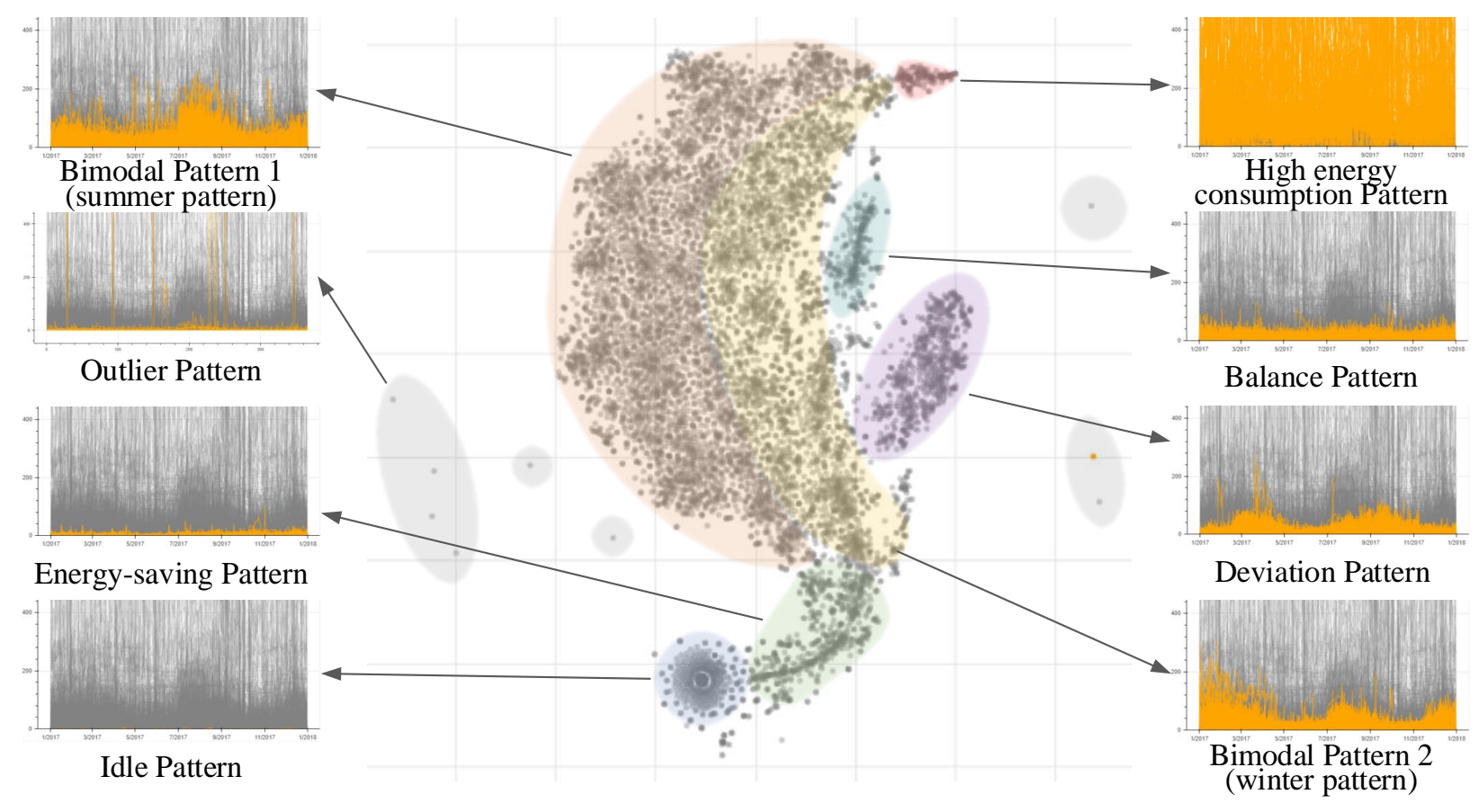

Figure 3: Cluster analysis of Shanghai households in 2017.

from the bimodal pattern, the peak periods are more concentrated in late winter and late summer. Furthermore, the idle pattern also accounts for a considerable proportion, due to the large number of vacant houses in Shanghai. The outlier pattern may have been caused by equipment failure, human error, or power theft. These data were therefore excluded as outliers from the energy demand forecast calculations.

\subsubsection{Prediction of energy demand}

Feature engineering. Energy demand is usually predicted based on a time series prediction algorithm and energy related features such as temperature, humidity, energy price, etc [54]. In this paper, we collected 15 weather features for the energy consumption forecasting process. The numerical features were: (1) daily low temperature (LT), (2) daily high temperature (HT), (3) daily low dew temperature (LDT), (4) daily high dew temperature (DHT), (5) daily low humidity (LH), (6) daily high humidity (HH), (7) daily low wind speed (LW), (8) daily high wind speed (HW), (9) daily low air pressure (LA), (10) daily high air pressure (HA), and (11) air pollution index (AP). The categorical features were (12) weather (WT), (13) wind direction (WD), (14) weekday (Wd), and (15) holiday index (HI). Among them, weather included light rain, clouds, sleet, and 11 other typical kinds of weather conditions. The holiday index was the number of vacation days, usually ranging from 1 to 8 (workdays were 0). However, the Spring Festival has special significance for Chinese people (most people leave the city and go back to the countryside during the Spring Festival), so it was coded separately, as 9.

Since the value range of the raw data varied, the value range of all numerical features needed to be normalized 
so that each characteristic contributed approximately proportionally to the forecasting model [55]. For categorical features, a word embedding technique was applied in this work, to meaningfully represent categories in the embedded space.

Deep network structure Then, this paper uses the encoding-decoding based long-term memory neural network to predict the future energy consumption. The encoder-decoder model can learn the meaning of each element from the input sequence and provide the decoder with information about the hidden state of each encoder [52]. This allows the model to focus on useful parts of the input sequence.

For time series forecasting, given the input sequence $\tilde{x}_{t}=\left(\tilde{x}_{1}, \tilde{x}_{2}, \ldots, \tilde{x}_{T}\right)$, the encoder can be applied to learn a mapping from $\tilde{x}_{t}$ to $h_{t}$ (at time step $t$ ) with:

$$
h_{t}=\operatorname{LSTM}\left(h_{t-1}, \tilde{x}_{t}\right)
$$

Where $h_{t} \in R^{m}$ is the hidden state of the encoder at time $\mathrm{t}, x_{t}$ is 15 weather features, $t \in[1,15], m$ is the size of the hidden state, and $\operatorname{LSTM}(x)$ is the non-linear activation function for capturing long-term dependencies. The structure of an LSTM cell is shown in figure 4

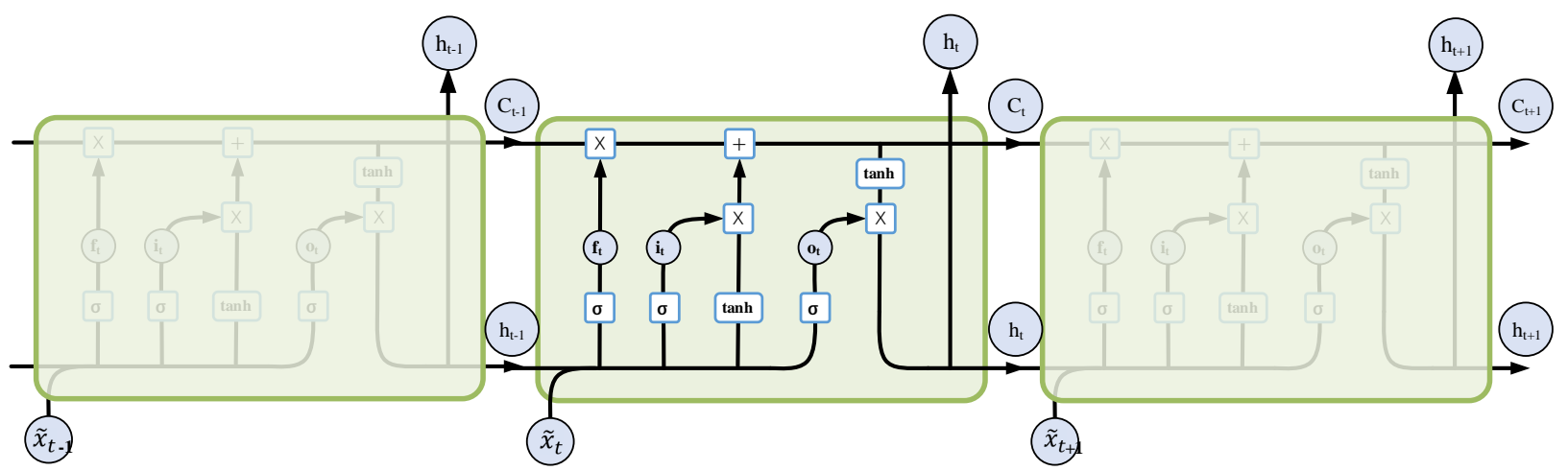

Figure 4: Structure of the LSTM.

Each LSTM unit has a memory cell with the cell-state $c_{t}$ at time t. Three sigmoid gates control access to the memory cell: forget gate $f_{t}$, input gate $i_{t}$, and output gate $o_{t}$. The following equations express the computational 
process of a single LSTM:

$$
\begin{aligned}
f_{t} & =\delta\left(W_{f}\left[h_{t-1} ; \widetilde{x_{t}}\right]+b_{f}\right) \\
i_{t} & =\delta\left(W_{i}\left[h_{t-1} ; \widetilde{x_{t}}\right]+b_{i}\right) \\
o_{t} & =\delta\left(W_{o}\left[h_{t-1} ; \widetilde{x_{t}}\right]+b_{o}\right) \\
\widetilde{c_{t}} & =\tanh \left(W_{c}\left[h_{t-1} ; \widetilde{x_{t}}\right]+b_{c}\right) \\
c_{t} & =f_{t} \times c_{t-1}+i_{t} \times \widetilde{c_{t}} \\
h_{t} & =o_{t} \times \tanh \left(c_{t}\right)
\end{aligned}
$$

Where $\tilde{x}_{t}$ is the input at time $\mathrm{t}, h_{t}$ is the hidden state at time $t . i_{t}, f_{t}$ and $o_{t}$ represent the input gate, forget gate, and output gate, respectively; $c_{t}$ is the cell state; $\tilde{c}_{t}$ is the candidate cell state; and $W_{f}, W_{i}, W_{o}, W_{c} \in R^{m(m+n)}$ and $b_{f}, b_{i}, b_{o}, b_{c} \in R^{m}$ are the parameters to learn. The key reason for using an LSTM unit is, that the cell state sums activities over time, which can overcome the problem of vanishing gradients and better captures the long-term dependencies of time series data.

To predict the output $y_{n}$, we used another LSTM neural network to decode the encoded input information. Similar to the encoder process, LSTM computes a mapping from an input dataset $C_{n}^{\prime}$ to an output series $h_{t}^{\prime}$ with three different gates. The following equation expresses the mapping process:

$$
h_{t}^{\prime}=\operatorname{LSTM}\left(h_{t-1}^{\prime}, C_{n}^{\prime}\right)
$$

In the equation above, $h_{t}^{\prime}$ can be updated as:

$$
\begin{aligned}
f_{t}^{\prime} & =\delta\left(W_{f}^{\prime}\left[h_{t-1}^{\prime} ; C_{n}^{\prime}\right]+b_{f}^{\prime}\right) \\
i_{t}^{\prime} & =\delta\left(W_{i}^{\prime}\left[h_{t-1}^{\prime} ; C_{n}^{\prime}\right]+b_{i}^{\prime}\right) \\
o_{t}^{\prime} & =\delta\left(W_{o}^{\prime}\left[h_{t-1}^{\prime} ; C_{n}^{\prime}\right]+b_{o}^{\prime}\right) \\
\widetilde{c_{t}^{\prime}} & =\tanh \left(W_{c}^{\prime}\left[h_{t-1}^{\prime} ; C_{n}^{\prime}\right]+b_{c}^{\prime}\right) \\
c_{t}^{\prime} & =f_{t}^{\prime} \times c_{t-1}^{\prime}+i_{t}^{\prime} \times \widetilde{c_{t}^{\prime}} \\
h_{t}^{\prime} & =o_{t} \times \tanh \left(c_{t}^{\prime}\right)
\end{aligned}
$$

where $\left[h_{t-1}^{\prime} ; C_{n}^{\prime}\right]$ is a concatenation of the hidden state $h_{t-1}^{\prime}$ and the decoder input $C_{n}^{\prime} . W_{f}^{\prime}, W_{i}^{\prime}, W_{o}^{\prime}, W_{c}^{\prime}$ and $b_{f}^{\prime}, b_{i}^{\prime}$, $b_{o}^{\prime}, b_{c}^{\prime}$ are parameters to be trained; and $\delta(x)$ and $\tanh (x)$ are a logistic sigmoid function and hyperbolic function, respectively.

$$
\begin{aligned}
y_{n} & =F\left(y_{1}, \ldots, y_{n-1}, x_{1}, \ldots, x_{T}\right) \\
& =v_{y}^{T}\left(W_{y}\left[h_{n}^{\prime} ; C_{n}^{\prime}\right]+b_{w}\right)+b_{v}
\end{aligned}
$$


where $\left[h_{n}^{\prime} ; C_{n}^{\prime}\right]$ is a concatenation of the hidden decoder state and the connection vector. The parameters $W_{y}$ and $b_{w}$ map the concatenation to the size of the hidden decoder states. The linear function with weights $v_{y}^{T}$ and $b_{v}$ produces the final forecasting result.

Output of the predicted results In this paper, the LSTM algorithm will be executed twice. First, the LSTM model is implemented for each electricity consumption pattern, and the prediction results of each pattern are added together as the total power demand of Shanghai. According to the theoretical analysis in literature [51] and the experimental results in this paper, the prediction result is superior to other results without clustering.

Then, to predict the spatial fluctuations of the energy demand, the future electricity demand of every household needs to be predicted. To balance the model resolution with data availability and computational tractability, only the future energy consumption of energy-intensive households are predicted by the LSTM. In this study, energy-intensive households were determined by using a filter coefficient (see section 3.4). The prediction results will be transmitted to the fusion module for spatio-temporal modeling, as shown in figure 1 .

\subsection{Fusion of spatial information}

When we regard the intensity of the energy demand as the spatial density, the density map can be used to visualize the spatial distribution of the energy demand. In this paper, the density map is obtained by using a kernel density estimation method (KDE), so that the discrete spatial energy information can be transformed into a continuous function for further analysis. The method is formalized in the following:

Let $x_{1}, x_{2}, \ldots, x_{n}$ be the discrete geographical locations of the households, $x_{i}$ denoted as a vector $\left(\operatorname{lon}_{i}, \text { lat }_{i}\right)^{\tau}$ of longitude and latitude. For time $t$, the density of a position of $\mathrm{x}$ in a density map is defined as follows:

$$
\left.\hat{f}_{t}(x)\right|_{t}=\frac{1}{n} \sum_{i=1}^{n} c_{i} K_{h}\left(x-x_{i}\right)=\frac{1}{n h} \sum_{i=1}^{n} c_{i} K \frac{x-x_{i}}{h}
$$

where $n$ is the sample size, $h$ is the bandwidth of the kernel $K_{h}$, which is symmetrical and positive definite, and $c_{i}$ is a weight coefficient of the energy consumption used to re-weight the demand strength with respect to the geographical distribution, which can be expressed with the following formula:

$$
c_{i}=\left\lfloor\frac{E}{c o e}\right\rfloor
$$

Where, $E$ represents the energy consumption of $x_{i}$, and coe is the filter coefficient defined by the power plant operator. When the filter coefficient is large, more consumers will be eliminated due to the round off function, the calculation load of KDE and the model resolution are both low; When the filter coefficient is small, more samples will be retained, which will lead to a higher model resolution and higher calculation costs. The consumers participating in the forecasting process in this paper account for about $10 \%$ of the total number of consumers. Their electricity consumption accounts for about $51 \%$ of the total. In addition, since the Gaussian kernel can capture data sensitivity of a large spatial area, and it has a lower computation complexity compared with other kernels with exponential 
functions, it is here used for the implementation. The original energy data and the optimization results of KDE are shown in the figure 5. The original data are represented by a 3-D histogram, and the optimized data of KDE are represented by a thermal diagram.

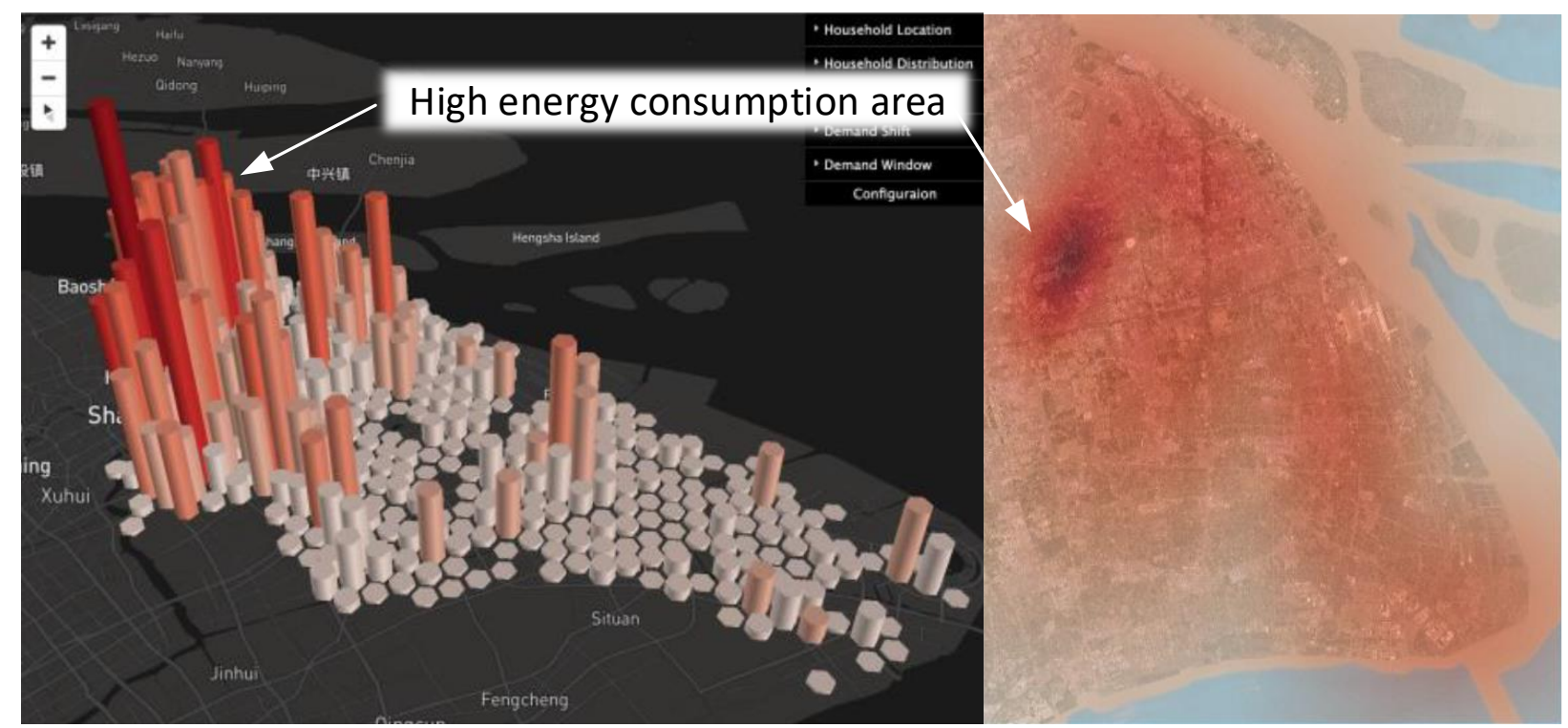

Figure 5: Distribution of power consumption in Shanghai before(left) and after(right) KDE.

Based on the latitude and longitude information of the consumers, the KDE method processes the historical and the predicted energy consumption data respectively to obtain the historical and the future KDE distribution. The predicted energy consumption data is derived from the energy information prediction module (refer to 3.3.2).

\subsection{Spatio-temporal demand-shift modeling based on vector field}

In this section, we describe our potential-flow-based modeling method. From the previous section, the spatiotemporal distributions of the discrete energy demand are expressed as two different density-strength maps. We observed, that the geospatial energy demands fluctuate continuously over time, and thus, the energy demand is a continuum occupying a simply-connected region in the time dimension with a irrotational characteristic. Therefore, the potential-flow method [56] can be used to visualize the spatial shift of high-energy demand flow, which implies the flow between spatially different areas.

Formally, the spatio-temporal demand-shift is defined as $\left.\nabla \operatorname{shift}(x)\right|_{t_{1}, t_{2}}$, which is the gradient of the velocity potential $\operatorname{shift}(x)$ that refers to the spatial energy demand fluctuation at the selected moments or periods.

$$
\left.\operatorname{shift}(x)\right|_{t_{1}, t_{2}}=\left.\hat{f}_{t}(x)\right|_{t_{2}}-\left.\hat{f}_{t}(x)\right|_{t_{1}}
$$

Where, $\hat{f}_{t}(x)$ is the result of KDE in equation 11 , namely the energy consumption distribution. $x$ denoted as a vector $(\text { lon, } l a t)^{\tau}$ of longitude and latitude. $t 2$ represents a time period to be predicted in the future, and $t 1$ represents 
a time period in the past with the same time span as $t 2$. The visual representation of formula 13 is shown in figure 6
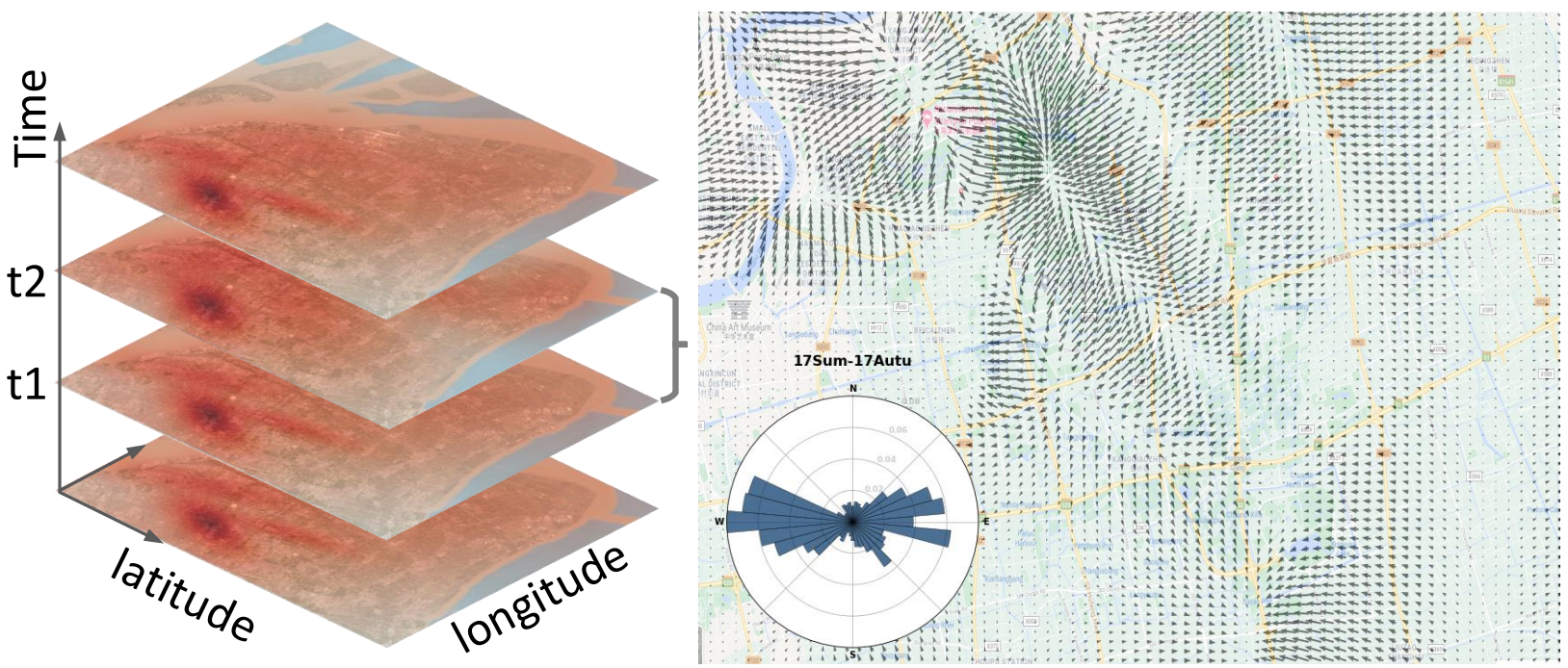

Figure 6: Principle of the vector field map.

The vector field map in figure 6 6 represents the spatio-temporal demand-shift model in the future compared with a certain period in the past. The vectors in the vector field map point to the direction of the shift in energy demand, and the magnitude of a vector represents the intensity of the shift. Therefore, this potential flow field can be defined as "Energy Demand Flow" from the perspective of energy consumption. The significance of the energy demand flow lies in the spatial and temporal distribution of energy consumption fluctuations (note: not the distribution of energy consumption). After we obtained the vector field, we can further extract the direction information of the energy demand flow. Specifically, for every flow field map, we divide all of the vectors into 36 equal-sized bins (i.e., each bin represents $10^{\circ}$ ) and use the polar coordinate histogram to visualize it, as shown in the polar coordinate diagram at the lower left corner of the flow field map in figure 6 Once the bearings are binned, we calculate the Shannon entropy $H$ as a measure of spatio-temporal demand-shift, as:

$$
H=-\sum_{i=1}^{n} P\left(o_{i}\right) \log _{e} P\left(o_{i}\right)
$$

Where $n$ represents the total number of bins, $i$ indexes the bins from 1 to 36 , and $P\left(o_{i}\right)$ represents the proportion of orientations that fall in the $i_{t h}$ bin. Similar to "Energy Demand Flow", the entropy in formula 14 can be defined as "Energy Demand Entropy". The meaning of energy demand entropy lies in the degree of chaos of the energy demand shifts (note: not the degree of chaos of the energy demand).

\section{Evaluation}

Since the spatio-temporal prediction in this paper is based on time series prediction, this section examines the effectiveness and robustness of the clustering-based LSTM introduced in 3.3.2. In addition, to investigate the superi- 
ority of the proposed model in power consumption forecasting, the results and other machine learning models were compared. The results are compared and discussed below.

\subsection{Experimental settings}

Datasets. All energy data used for this study are derived from the Shanghai Power Bureau, including electrical energy consumption data for three years of approximately 10,000 households in Shanghai. The dataset is usually dailygrained with peak and valley detailed time-series data. Meteorological parameters are obtained from the public dataset of the National Meteorological Information Center, China Meteorological Administration. The energy consumption data in 2017 were used to train the network parameters, and the energy data for 2018 were used as a test dataset to evaluate the model's performance and prediction ability. As mentioned above, the weather is the main factor affecting short-term power consumption. Therefore, 15 weather parameters were selected as features for model training.

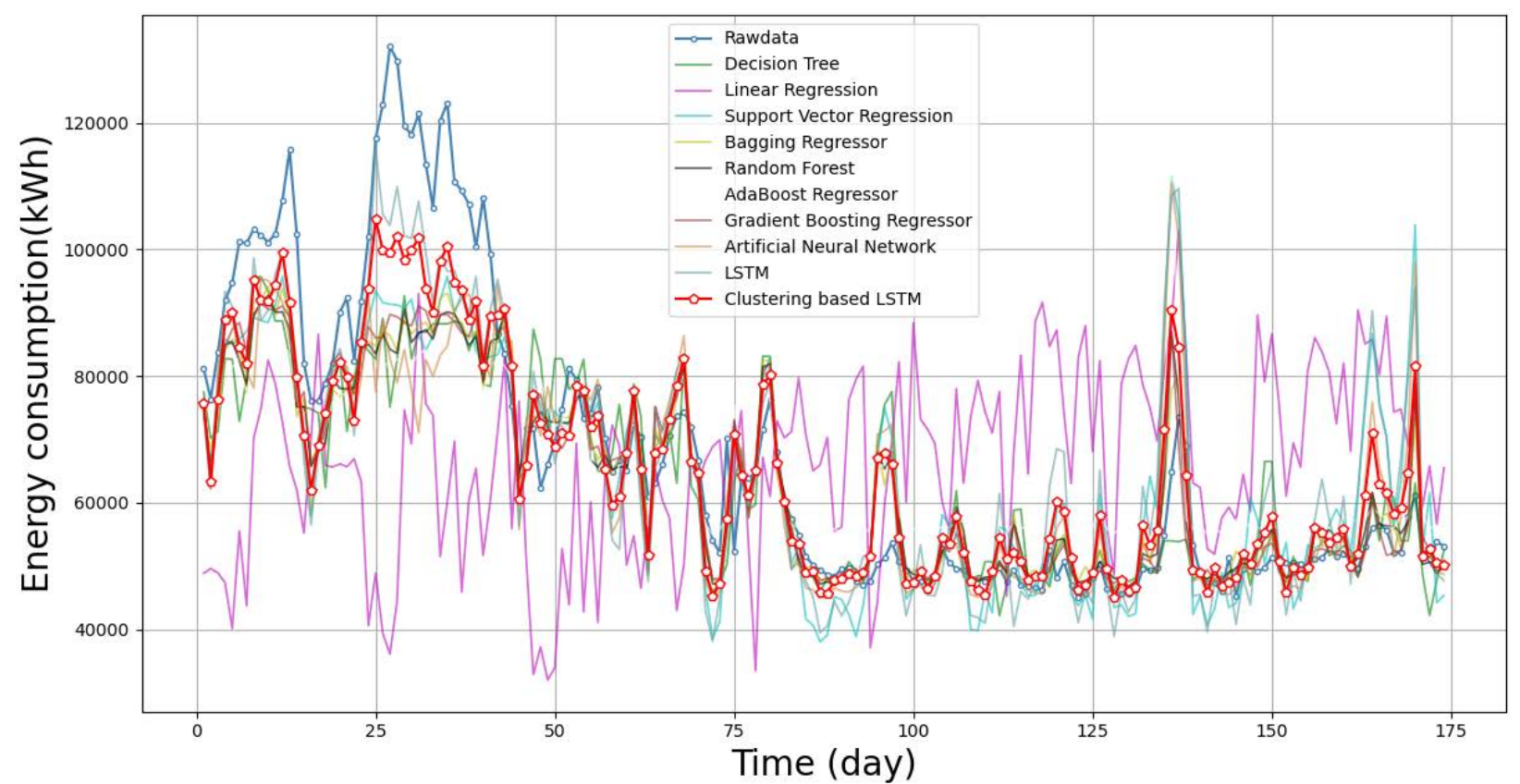

Figure 7: Prediction results from different machine learning models.

Comparison with classic approaches. In the experiments, we employed 7 classic machine learning algorithms and 2 neural network based algorithms as the baseline for the comparison of energy forecast results, among which machine learning methods [59] include: decision tree, linear regression, support vector regression, bagging regressor, random forest, AdaBoost regressor, gradient boosting regressor, while neural network based methods include: artificial neural network [60] and LSTM [61].

In order to measure the effectiveness of different algorithms uniformly, all samples and features are involved in each training batch (max samples=1, max features=1) for the training of traditional regression algorithms (DT, LR, SVR, BR, RF, ABR, and GBR) and the number of estimators is all 100. In particular, the loss functions of RF and 
GBR are cross-entropy; MSE is used to judge whether the node should continue splitting; The splitting stops when the number of samples of a node is less than 2. For both artificial neural networks and LSTM, the activation function is tanh; Adam optimizer is used for gradient descending; The loss function is MSE; the batch size and epochs are set to 64,100 respectively. The artificial neural network contains two hidden layers, each with 100 neurons, while the LSTM uses a single hidden layer structure with 50 units in the hidden layer.

Further we use our proposed clustering-based LSTM, in which we apply an LSTM to each energy consumption pattern respectively. The predicted values of different patterns are added together as the total energy consumption of Shanghai. Then, the prediction accuracy of clustering based LSTM is compared with other methods.

Parameters and evaluation metrics. For the clustering based LSTM an Adam optimizer [62] was also used to train our model. According to the literature[63] and experimental results, the batch size and epochs were set to 128 and 5,000, respectively. To avoid gradient explosion, we set the maximum gradient to 2 ; the dropout rate for each LSTM function was set to 0.3 to avoid over-fitting. To quantify the performances of the models, we applied three indicators: Root Mean Square Error(RMSE), Mean Absolute Error(MAE), Mean Square Logarithmic Error(MSLE), $\mathrm{R}$ squared $\left(R^{2}\right)$ [64]. These indicators can be defined as follows:

$$
\begin{aligned}
R M S E & =\sqrt{\frac{1}{N} \sum_{i=1}^{N}\left(y_{i}{ }^{\prime}-y_{i}\right)^{2}} \\
M A E & =\frac{1}{N} \sum_{i=1}^{N}\left|y_{i}{ }^{\prime}-y_{i}\right| \\
M S L E & =\frac{1}{N} \sum_{i=1}^{N}\left|\log \left(y_{i}+1\right)-\log \left(y_{i}{ }^{\prime}+1\right)\right|^{2} \\
R^{2} & =1-\frac{\sum_{i=1}^{N}\left(y_{i}-y_{i}{ }^{\prime}\right)^{2}}{\sum_{i=1}^{N}\left(y_{i}-\bar{y}\right)^{2}}
\end{aligned}
$$

where $N$ is the length of the time series, $y_{i}$ is the actual data, and $y_{i}{ }^{\prime}$ is the predicted value.

\subsection{Experimental results}

For the real-life data-sets, the prediction results of the LSTM algorithm in different patterns are shown in figure 7 , and the prediction accuracy of all methods are shown in table 1 and table 2 The clustering based algorithm proposed in this paper is essentially an ensemble learning method based on stacking, which uses multiple learning algorithms to obtain a better predictive performance than could be obtained from any of the constituent learning algorithms alone [58].

According to the MSLE in table 1, the estimation errors of the idle pattern and energy-saving pattern are the highest, which are 0.57 and 0.55 , respectively. This result can be attributed to the existence of a large majority of zeros in the energy data (labels) of these two patterns. Fortunately, the energy consumption of these two patterns 
Table 1: Prediction results of LSTM algorithm in different patterns

\begin{tabular}{ccccc}
\hline & RMSE & MAE & MSLE & R2 \\
\hline Balance pattern & 248.71 & 209.79 & 0.01 & 0.42 \\
Bimodal pattern-deviation & 116.71 & 85.86 & 0.17 & -3.40 \\
Bimodal pattern-winter & 4177.73 & 3119.53 & 0.02 & 0.88 \\
Bimodal pattern-summer & 7963.82 & 5860.95 & 0.09 & 0.09 \\
High energy consumption pattern & 2131.48 & 1829.74 & 0.03 & -0.13 \\
Idle pattern & 40.73 & 33.54 & 0.57 & -1.99 \\
Energy saving pattern & 398.92 & 374.60 & 0.55 & -5.63 \\
\hline
\end{tabular}

accounts for a tiny proportion of the total. For example, the energy consumption of the idle pattern and the energysaving pattern accounted for $0.0033 \%$ and $0.54 \%$ of total energy consumption in 2017 , respectively. Therefore, the impact of the two patterns on the total prediction error is limited. In addition, LSTM performs best in the balanced pattern due to the stable energy consumption. From table 2, we can see that the clustering-based LSTM, in general, has fewer errors than other approaches. This can be attributed to each pattern having a higher signal-to-noise ratio after clustering, due to the aggregation.

Different from the prediction error described by MSLE, R2 is a statistical measure that represents the goodness of fit of a regression model. In Table 2, linear regression has the lowest R2 and the highest RMSE. This is due to the fact that linear regression assumes a linear relationship between dependent and independent variables, which makes it difficult to describe the nonlinear dependencies in energy consumption. For the different energy consumption patterns in Table 1, R2 is negative for the high energy consumption pattern, idle pattern, and energy-saving pattern, which can be explained by the high randomness of the electricity consumption habits of the customers in these patterns. Here, the higher randomness means that the power consumption is less associated with the weather parameters. Specifically, customers in the high energy consumption pattern usually consume electricity based on their individual requirements, rather than on external factors (excessively high or low temperatures, uncomfortable humidity, etc.). In addition, the use of high-powered appliances also enhances the randomness of energy consumption. Customers in low energy consumption pattern consume electricity only for the basic necessities of life and do not use air conditioning and heating equipment indiscriminately. The idle pattern usually refers to a vacant house with no electricity consumption, where energy consumption is not related to weather factors at all.

\section{Visualization interface and case study}

In this section, we describe the visual interface and some cases of the interaction system. The visual analysis process is implemented as a web application and can be applied to any kind of energy such as natural gas, solar energy, etc. This research uses the electric energy of Shanghai as an example for the analysis. As figure 8 shows, the system consists of four coordinated interfaces, which are pattern discovery (PD), time series prediction (PP and 
Table 2: Comparison of the total energy consumption prediction results of clustering based LSTM with other traditional methods

\begin{tabular}{ccccc}
\hline & RMSE & MAE & MSLE & R2 \\
\hline Decision Tree & 12986.78 & 8301.78 & 0.024 & 0.67 \\
Linear Regression & 30208.06 & 25203.71 & 0.194 & -0.79 \\
Support Vector Regression & 13522.85 & 9881.47 & 0.031 & 0.64 \\
Bagging Regressor & 11425.61 & 7204.18 & 0.017 & 0.71 \\
Random Forest & 11935.21 & 7535.36 & 0.019 & 0.72 \\
AdaBoost Regressor & 14487.82 & 10531.54 & 0.031 & 0.59 \\
Gradient Boosting Regressor & 11302.68 & 7008.19 & 0.017 & 0.71 \\
Artificial Neural Network & 14057.15 & 8761.12 & 0.027 & 0.61 \\
LSTM & 11879.03 & 8828.73 & 0.027 & 0.72 \\
Clustering based LSTM & 9446.15 & 6741.18 & 0.014 & 0.83 \\
\hline
\end{tabular}

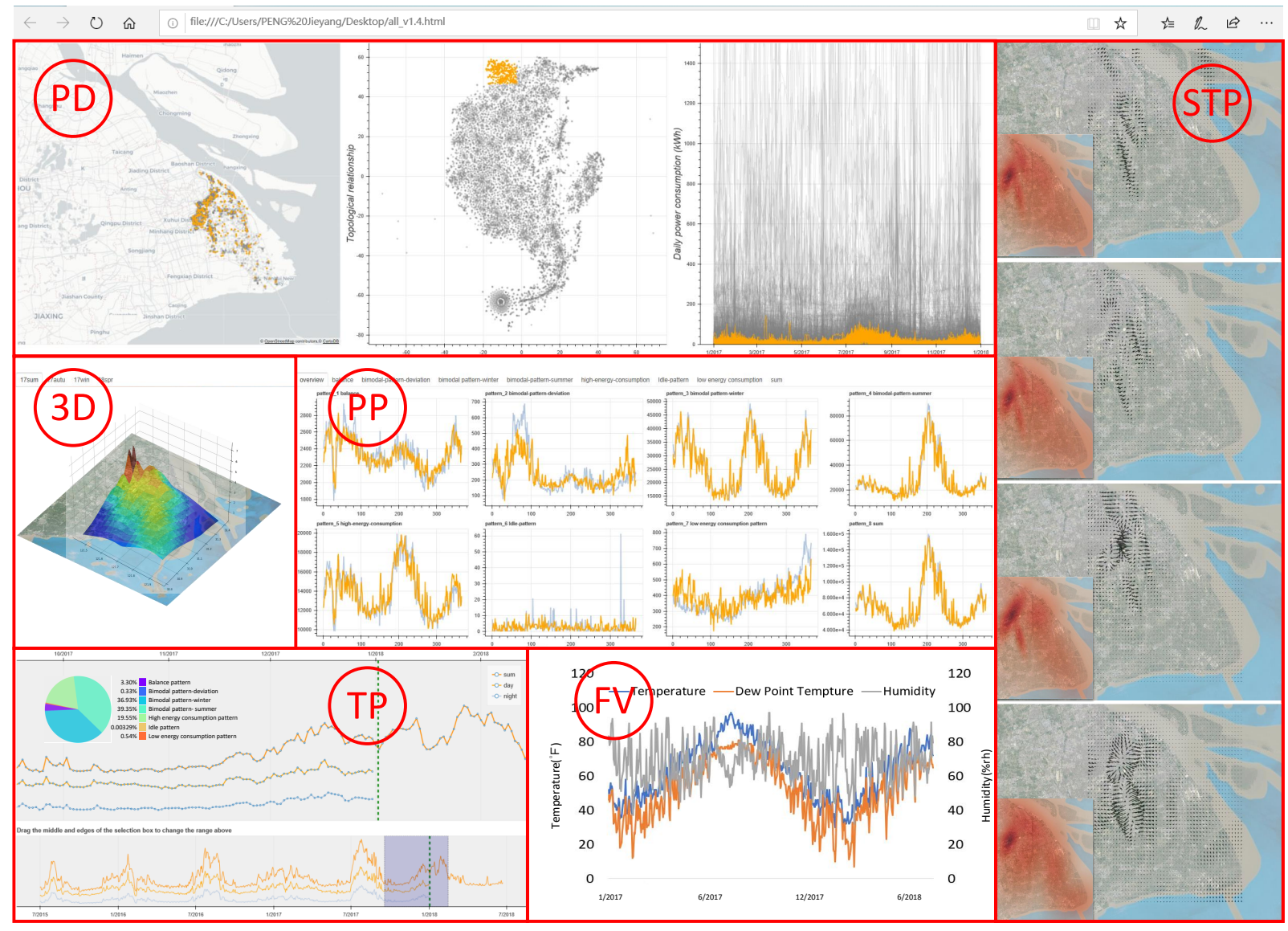

Figure 8: Visual analysis interface for temporal and spatial analysis. The interface includes consumption pattern discovery, spatio-temporal energy model, time series prediction, and spatio-temporal prediction. PD=Pattern discovery; $\mathrm{PP}=\mathrm{Pattern}$ prediction; $\mathrm{TP}=$ Time series prediction; $\mathrm{STP}=$ Spatio-temporal prediction; 3D=3D Model; FV=Feature visualization. 
TP), spatio-temporal prediction (STP), and feature visualization (3D and FV). This example is based on the power consumption data of Shanghai in 2017 and is used to forecast the spatio-temporal energy demand in 2018.

\subsection{Pattern discovery (PD)}

Figure 8 shows the main interface of the pattern discovery module (PD), which consists of the three views: a geography map (left), a topology map (middle) and a time series energy consumption diagram (right), which are linked by the backend database.

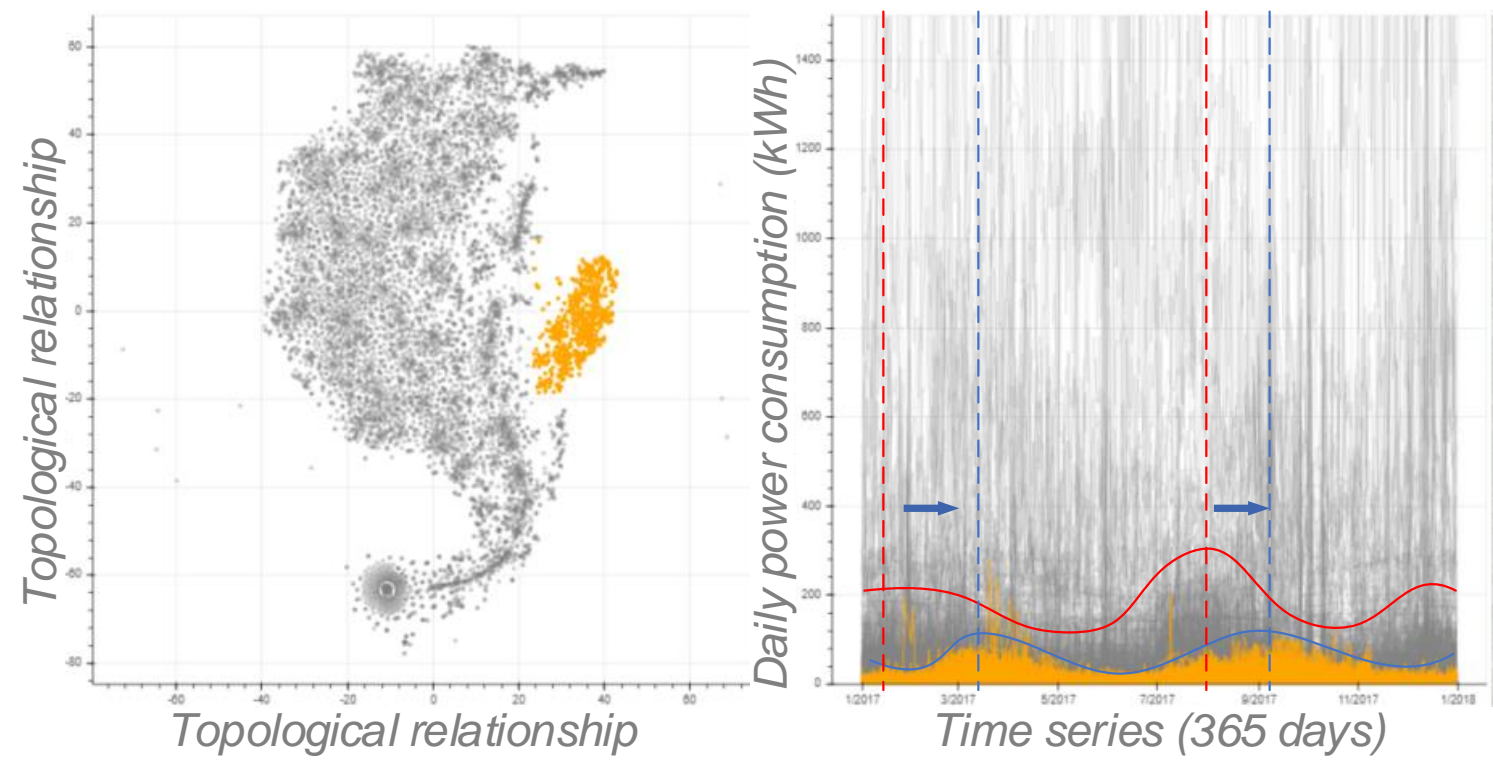

Figure 9: Comparison between the deviation pattern and the bimodal pattern.

The topology map is based on the t-SNE clustering of the customer's electricity usage (refer to section 3.3.1). With the reduced data points, typical patterns of energy consumption can be interactively identified by selecting the closely placed points on the view in a low-dimensional space. The closer the points are to each other, the more similar the patterns will be. The selected customers' geographical distribution and energy consumption data will be displayed on the geographic map (left) and the time-series diagram (right), respectively. Figure 3 shows a typical classification of energy consumption patterns. Among them, we found a special pattern, namely the deviation pattern, as shown in figure 9. As a subspecies of the bimodal pattern, the peak power consumption of the deviation pattern appears in September and March, which is more than a one month delay from the peak period (August and January) of the traditional bimodal pattern. Although the overall energy consumption may not necessarily be reduced, studying the cause of the deviation pattern is helpful to promote peak load shifting and reduce the peak load in power plants. 
The geographic map displays the spatial information of the clustered customers in the topology map. Similar to the topology map, customers can select points in the geographic map and observe the pattern distribution and energy consumption data of customers represented by these points in the topological map and the time series diagram. As shown in figure 10, we selected four regions in Shanghai and observed the power consumption in these regions. The two areas on the left in figure 10 correspond to the Lujiazui Financial Center (lower left) and the Waigaoqiao Port (upper left) respectively. The Lujiazui Financial Center, as one of the busiest districts of Shanghai also shows the highest amount of electricity consumption. The two areas on the right are far from the center of Shanghai, where agriculture is the dominant economy, corresponding to a relatively low consumption of electricity.
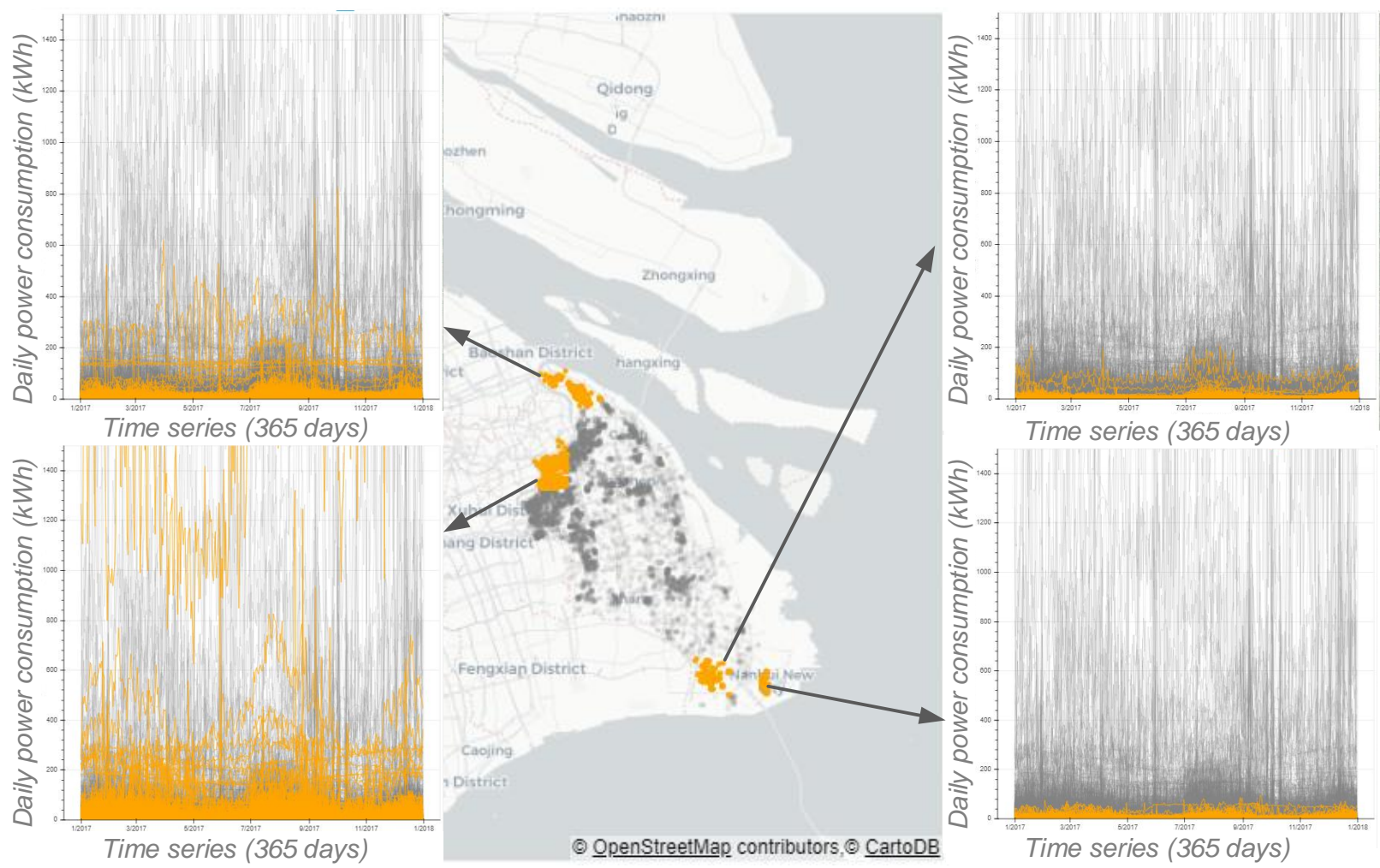

Figure 10: Energy consumption pattern discovery based on geography map.

\subsection{Time series prediction}

The time series prediction module is mainly used to display the composition and prediction results of urban total energy consumption.

Pattern prediction (PP). As described in 3.3 .2 , the power consumption prediction results of each pattern were displayed on the pattern prediction (PP) interface. In the PP interface, we can observe the prediction effect of all patterns at the same time, and we can also investigate the prediction process of a certain pattern in detail through the control panel, as shown in figure 11 . 


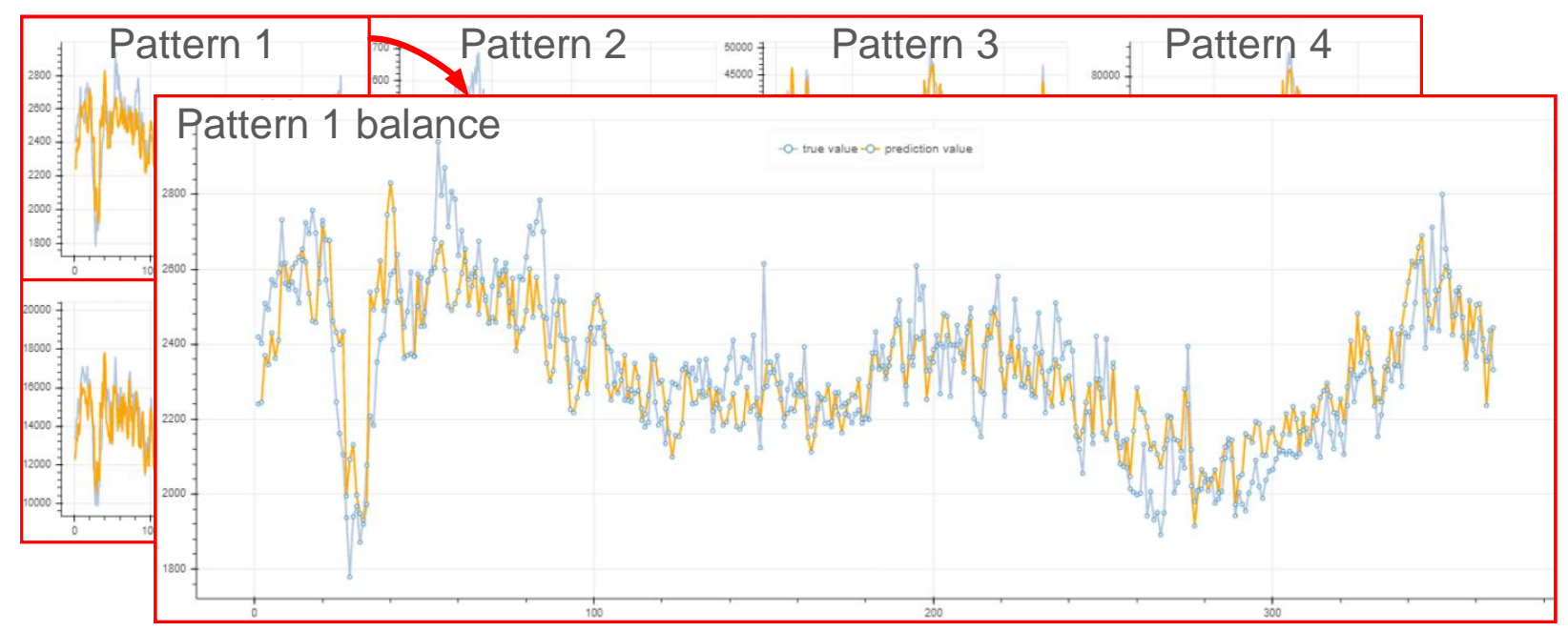

Figure 11: Time series prediction of energy demand in different patterns.

Time series prediction (TP). In the time series prediction interface, the prediction results of all patterns are added together as the total urban energy consumption data. In the TP interface shown in figure 8 , the line chart below is a panoramic view, recording the total energy consumption data of the city since the system was applied in July 2015. The left side of the dotted green line is the peak (6:00 22:00), valley (22:00 6:00) and total value of urban daily electricity consumption, while the right side represents the predicted value of electricity consumption. By dragging the purple box in the panoramic view, we can observe the energy data in the box in detail in the line chart above.

\subsection{Spatio-temporal prediction (STP)}

The spatio-temporal prediction module is used to predict the spatial variation of the energy consumption in the future, which can be visualized by a vector field. The four-vector field maps define the energy demand flow from spring 2017 to summer 2017, summer 2017 to autumn 2017, autumn 2017 to winter 2017, and winter 2017 to spring 2018, respectively. The energy statistics for 2017 are historical data, and the data for 2018 are predicted data. Therefore, the vector field map (lower right) is the consequence of the spatio-temporal prediction from winter 2017 to spring 2018. In order to obtain the spatio-temporal prediction results with high spatial resolution, we can also construct a vector field model for a specific region in the city. Further, different shifting patterns can be discovered by varying the temporal granular intervals, including hourly, every four hours, daily, weekly, monthly, quarterly, and yearly, so that the sensitivity of the shift pattern changes against different time granularity can be learned. With this, an appropriate advice can be given for the short-term operation and planning of power plants.

For example, figure 12 shows the energy spatial variation in the Lujiazui area from peak period $t 2$ (6:00-22:00) to valley period $t 1$ (22:00-6:00). It should be noted that the time span of the peak period and the valley period is different, so that the energy data needs to be normalized before the KDE optimization. The source area of arrows represents a commercial area, while the area pointed to by the arrows is a residential area (i.e. the light orange area). 
This flow map indicates that the area with high energy demand is shifted to the residential area when people go home after work.

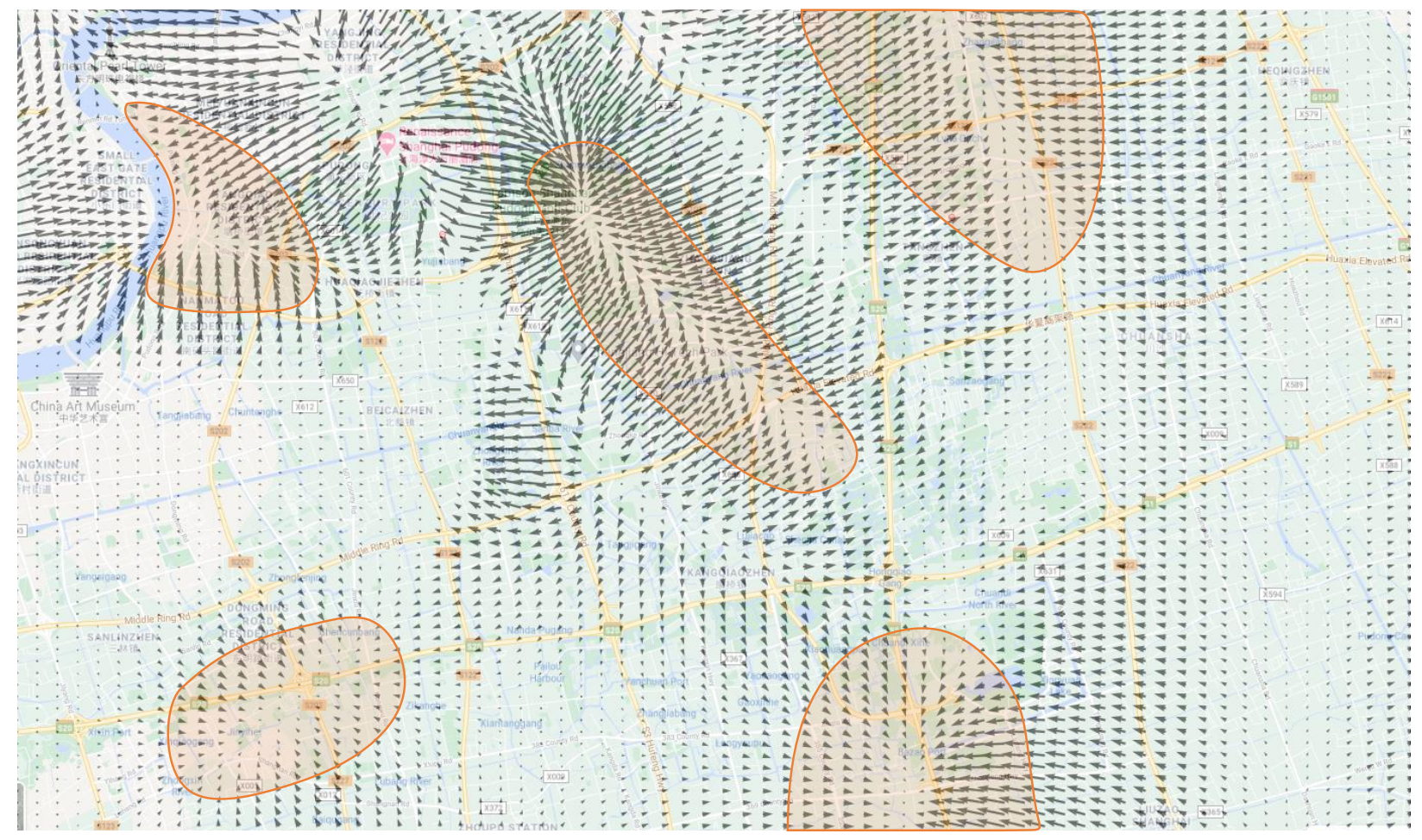

Figure 12: Spatial shift of energy from peak(6:00-22:00) to valley(22:00-6:00). periods.

In addition, the spatial shift pattern affected by different customer groups according to the consumption intensity is also worth analyzing. We can learn the sensitivity against different energy consumption intensities by selecting different customer groups. For example, figure 13 shows the spatial shift of energy from winter to spring for customers with high energy consumption patterns and customers with energy saving patterns.

In our further analysis of the vector field, we found that, although the temperature in Shanghai is similar between May to June and September to October, the total energy demand in the later period is about 200,000 kWh higher. One of the reasons, we suspect, is the psychological inertia and potential energy efficiency response people can be expected to take [57]. Another notable conclusion is that energy demands are unbalanced between regions. The area in the Sanlin town (a highly dense residential area) has the largest spatial demand change (more than 80,000 kWh). This means that such an area may change the electricity consumption strongly. These areas of concern are defined as the "Energy Shift Vortices" in this paper. The light red area shown in figure 12 is also a typical Energy Shift Vortex, where the vectors are concentrated (compared to figure 12 . 

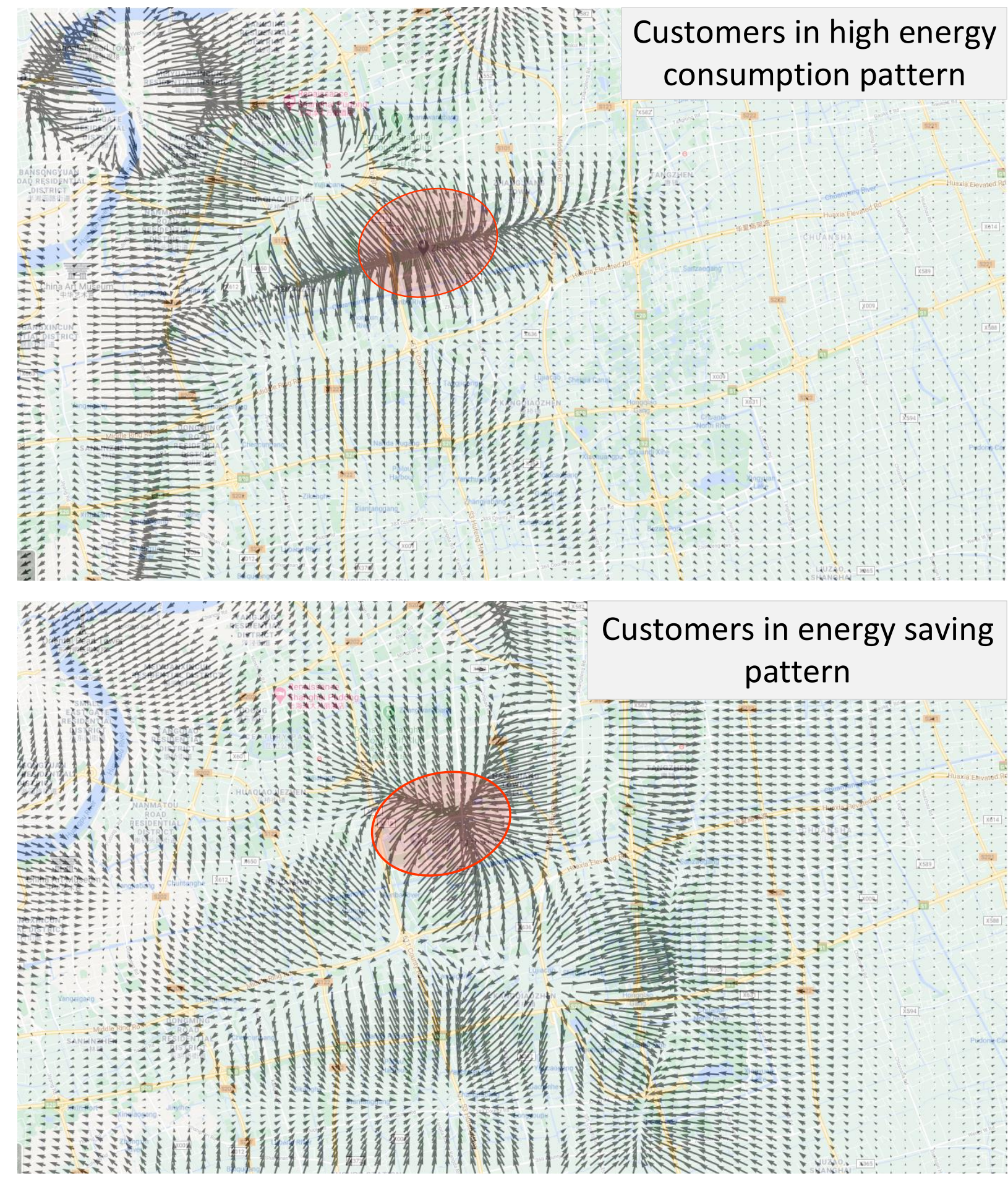

Figure 13: Spatial shift of energy from winter to spring for consumers with high energy consumption pattern(above) and energy saving pattern(below). 
Table 3: Statistics of energy demand entropy and meteorological data in 2016 and 2017. Energy consumption and meteorological data are the difference between the two quarters.

\begin{tabular}{|c|c|c|c|c|c|c|c|c|}
\hline & 15Win-16Spr & 16Spr-16Sum & 16Sum-16Autu & 16Autu-16Win & 16Win-17Spr & 17Spr-17Sum & 17Sum-17Autu & 17Autu-17Win \\
\hline Entropy & 3.42 & 3.47 & 3.23 & 3.47 & 3.26 & 3.28 & 3.33 & 3.46 \\
\hline Energy consumption $(k W H)$ & -26417.34 & 37765.01 & -36635.48 & 24212.71 & -19992.71 & 44299.74 & -44457.12 & 39242.92 \\
\hline Temperature $\left({ }^{\circ} F\right)$ & 17.88 & 21.63 & -15.51 & -21.70 & 16.29 & 21.95 & -17.51 & -24.76 \\
\hline Dew Point $\left({ }^{\circ} F\right)$ & 17.96 & 22.43 & -13.65 & -23.77 & 12.99 & 24.14 & -16.05 & -26.27 \\
\hline Humidity (\%) & 3.32 & 2.85 & 2.62 & -7.20 & -3.75 & 5.45 & 1.15 & -5.51 \\
\hline Wind Speed $(m p h)$ & 0.16 & 0.08 & -0.02 & -0.29 & 0.78 & -0.26 & 0.12 & -0.63 \\
\hline Pressure $(H g)$ & -0.35 & -0.28 & 0.33 & 0.27 & -0.31 & -0.27 & 0.35 & 0.23 \\
\hline Air Quality Index & -18.42 & -24.72 & -0.22 & 20.19 & -8.09 & -6.51 & -5.71 & 22.83 \\
\hline
\end{tabular}

\subsection{Polar histogram and energy demand entropy}

The polar histogram is used to further extract directional information of vector field diagrams (refer to 3.5 ). The polar histogram can be obtained by analyzing the magnitude and direction of all vectors in the vector field map. As the vector field is a materialized representation of the energy demand flow, the polar histogram reflects the trend of spatio-temporal shifts of energy (see figure 14). The four polar histograms in figure 14 are abstracted from the flow field of seasonal changes in spring of 2017 to spring of 2018. Due to the annual periodicity of energy shifts in time and space, we find that the change of the energy demand from spring to summer in Shanghai is mainly from north to south, while the change of energy demand from summer to autumn is mainly from east to west. Similarly, we can also further explore and anticipate the shift regularity of the energy demand flow in other seasons, months, or different periods of the day.

The energy demand entropy and meteorological data in 2016 and 2017 are shown in table 3 Among them, energy demand entropy is calculated by formula 14 . The energy data and the meteorological data are the difference between two quarters. In order to further study the relationship between the energy demand entropy, energy consumption, and meteorological data, this paper conducts a correlation analysis on the above data, as shown in table 4 It can be seen from table 4 that energy demand entropy is positively correlated with the fluctuation in energy consumption. This can be attributed to the fact that, at the urban level, the more dramatic the change in energy consumption, the more chaotic the demand for energy. In addition, the energy demand entropy has a weak negative correlation with wind speed and humidity. Therefore, extreme wind speed and humidity can stabilize fluctuations in energy consumption to some extent, although it has little effect on the total energy consumption.

\section{Discussion of policy implications}

Spatio-temporal peak-load shifting helps to reduce the maximum load on the power plant and optimize energy utilization. The traditional peak-load shifting is the process of mitigating the effects of large energy load blocks during a period of time by advancing or delaying their effects until the power supply system can readily accept 
Table 4: Correlation analysis of energy demand entropy, energy consumption and meteorological data

\begin{tabular}{|c|c|c|c|c|c|c|c|c|}
\hline & Entropy & Energy consumption $(k W H)$ & Temperature $\left({ }^{\circ} F\right)$ & Dew Point $\left({ }^{\circ} F\right)$ & Humidity (\%) & Wind Speed $(m p h)$ & Pressure $(\mathrm{Hg})$ & Air Quality Index \\
\hline Entropy & 1.00 & l & I & I & l & l & l & l \\
\hline Energy consumption $(k W H)$ & 0.51 & 1.00 & I & I & I & l & l & I \\
\hline Temperature $\left({ }^{\circ} \mathrm{F}\right)$ & -0.14 & 0.15 & 1.00 & I & I & l & I & I \\
\hline Dew Point $\left({ }^{\circ} F\right)$ & -0.16 & 0.14 & 1.00 & 1.00 & I & l & I & l \\
\hline Humidity (\%) & -0.39 & -0.16 & 0.59 & 0.66 & 1.00 & l & I & I \\
\hline Wind Speed $(m p h)$ & -0.45 & -0.59 & 0.51 & 0.48 & 0.15 & 1.00 & I & I \\
\hline Pressure $(\mathrm{Hg})$ & -0.01 & -0.23 & -0.96 & -0.94 & -0.40 & -0.47 & 1.00 & l \\
\hline Air Quality Index & 0.19 & 0.25 & -0.82 & -0.84 & -0.76 & -0.63 & 0.69 & 1.00 \\
\hline
\end{tabular}

additional load. Based on the empirical study of energy demand flows, urban energy demand shifting has a spatial concentration effect, which can be defined as the energy demand vortex. Consequently, the traditional temporal peakload shifting can be extended to the spatio-temporal domain. The intent behind the spatio-temporal peak-load shifting is to minimize generation capacity requirements by regulating load flow.

From a temporal perspective, regulating electricity prices is the easiest way to achieve peak-load shifting. For example, appropriately increasing the tariff in July and August to curb the peak energy consumption in summer, or reducing the tariff in the valley period, for example, at night. If the loads themselves cannot be regulated(for example, residents' rigid demand for cooling equipment in summer), this must be accomplished by implementing energy storage systems to shift the load profile. The energy storage systems is charged while the electrical supply system is powering minimal load and the cost of electric usage is reduced, e.g. at night. It is then discharged to provide additional power during periods of increased loading, while costs for using electricity are increased. This technique can be employed to mitigate utility bills. It also effectively shifts the impact of the load on the system, minimizing the generation capacity required.

From a spatial perspective, energy demand vortexes tend to appear in specific functional areas (e.g., the high-rise Lujiazui financial center and the densely populated Sanlin residential area), which can lead to high localized power loads. The energy needs of these vortexes are determined by regional functions and are usually hardly transferable. Possible solutions include providing incentives to the residents of the area for renewable energy in order to reduce their dependence on power plants, and to reduce the network loss during long-distance energy transmission. In addition, the adoption of a distributed urban planning contributes to avoiding the regional concentration effect of energy in a certain period of time, which means different functional areas should be uniformly distributed throughout the city, rather than in a centralized layout.

On top of the existing tiered tariff for electricity, a new tiered tariff should be added to reduce the living costs for customers in the energy-saving pattern. The current tiered pricing for electricity in Shanghai is shown in table 5 , As mentioned earlier, customers in the energy-saving pattern usually consume energy only to meet basic living needs. Therefore, their average electricity consumption can be considered as the minimum required to meet the local basic living, which suggests a rigid demand with high degree of price-inelasticity. In Shanghai, the average annual power 
Table 5: Tiered pricing for electricity in Shanghai

\begin{tabular}{cccc}
\hline Tariff & Power consumption & Peak/off-peak & Price \\
\hline Frist step & $0-3120$ & $\begin{array}{c}\text { peak period } \\
\text { off-peak period }\end{array}$ & $\begin{array}{c}0.617 \mathrm{yuan} / \mathrm{kWh} \\
0.307 \mathrm{yuan} / \mathrm{kWh}\end{array}$ \\
Second step & $3120-4800$ & $\begin{array}{c}\text { peak period } \\
\text { off-peak period }\end{array}$ & $\begin{array}{c}0.667 \mathrm{yuan} / \mathrm{kWh} \\
0.357 \mathrm{yuan} / \mathrm{kWh}\end{array}$ \\
& & $\begin{array}{c}\text { peak period } \\
\text { Third step }\end{array}$ & $0.917 \mathrm{yuan} / \mathrm{kWh}$ \\
& $>4800$ & off-peak period & $0.607 \mathrm{yuan} / \mathrm{kWh}$ \\
\hline
\end{tabular}

consumption of customers in the energy-saving pattern is $237 \mathrm{kWh}$. Considering that $237 \mathrm{kWh}$ is the average value of the energy-saving pattern, we recommend setting the new tiered price as $320 \mathrm{kWh}$ to cover the majority of customers of the energy-saving pattern. Figure 15 shows the histogram of the household demand distribution in Shanghai. The orange line in the figure is the existing tiered price, and the red area represents the customers of the idle pattern and the energy-saving pattern (as shown in the sub-figure).

The outlier pattern should be paid more attention to in further empirical studies of energy systems due to its strong correlation with equipment failure and power theft, although it is considered as abnormal data and eliminated during data preprocessing. In further research, a consumption pattern-based energy theft detector can be presented, which leverages the predictability property of customers' normal and malicious consumption patterns. Specifically, areas with a high probability of energy theft are short-listed, and by monitoring abnormalities in the consumption patterns, suspicious customers can be identified [65].

\section{Conclusions}

This paper presents the initial development of a spatio-temporal forecasting study that explores the future demand of daily energy consumption. The main contributions at this stage include:

- We provide a technique of clustering applied to energy data and analyzes how to reduce and visualize high dimensional energy data to low dimensional space. We discovered that the energy consumption pattern in Shanghai can be divided into eight typical patterns. As the most classic pattern of power consumption, the bimodal pattern has the largest number of customers. The bimodal pattern can be further divided into summer and winter patterns, which reflect the reliance of the Shanghai household on electricity to cope with hot summers and chilly winters. The data in the outlier pattern are excluded as outliers in this research, while in practical applications, the outlier pattern should be paid more attention to, as it may imply electricity meter failure or power theft. The future study of the deviation pattern is helpful to reduce the peak value of electricity consumption in summer and winter.

- Based on the clustering results, an electricity time series forecasting method is applied to predict the total power consumption in Shanghai. In the process of hierarchical forecasting, we found that classic machine 
learning algorithms presented a large error in predicting the idle pattern and the energy-saving pattern, which is caused by a large number of similar labels (power consumption is 0 ). Compared with other patterns, the customers' electricity consumption of these two patterns is also more random. For the idle pattern and the energy-saving pattern, autoregressive algorithms such as ARIMA(Autoregressive Integrated Moving Average) are more effective, because they do not rely on a large number of features. In addition, we also designed a comparative experiment including 7 classic machine learning algorithms and 2 deep learning algorithms. The experimental results show that the prediction accuracy of the clustering-based LSTM is higher, and the predicted value also has a stronger correlation with the real value.

- Based on the time series forecasting results, an innovative potential-flow model is created, which will generate better visualization of spatio-temporal energy demands in the future. The model supports multi-source and multi-granularity energy data. Furthermore, we propose the concept of "energy demand flow". Energy demand flow is an innovative visualization method and concept in the energy field, which can reflect the spatial change of energy demand over time. We also put forward the concept of "energy demand entropy", which can quantitatively describe the intensity of energy change in a given time span and geographical space.

- Based on the potential-flow model, We conducted an empirical study to analyze the sensitivity of the shift pattern changes against different time granularity and the shift pattern affected by different customer groups with real-life data in Shanghai. According to the potential-flow map, we found several cluster centers in Shanghai(for example, the Sanlin area mentioned in section 5.3), where the energy shift is quite intense. We define these areas as the "Energy Shift Vortex", which usually need to be focused on during seasonal variation or day/night shifts.

We provide a spatio-temporal analysis tool with an easily interpretable visualization of urban energy demand. Current literature has lacked such a systematic spatio-temporal demand characterization. Our system can make projections about the future status of the demand for various energy sources and their proportional relationships, which allows the government to establish the relationship between energy consumption and environmental protection and to adjust the energy structure accordingly. In summary, in contrast to traditional time-series energy data forecasting, this paper highlights the importance of realistically representing the spatial and temporal heterogeneity of urban demand in urban energy forecasting. The strength of local distribution changes, which mostly represents partial dynamics, is currently used to calculate the geographic shift of energy demand, but in fact, there is not only local dynamics but also a global trend. We would like to enhance the flow field generation algorithm in the future and deliver a more precise geographical demand shift.

\section{Acknowledgment}

The research is supported by National Key R\&D Program of China (No.2017YFE0101400), National Natural Science Foundation of China (No.61802278), China Scholarship Council, and EU H2020 Research and Innovation Program under the Marie Sklodowska-Curie Grant Agreement (754462). 


\section{References}

[1] Niu, Zhibin, et al. "Understanding energy demand behaviors through spatio-temporal smart meter data analysis.” Energy 226 (2021): 120493.

[2] Nose-Filho K, Lotufo ADP, Minussi CR. Short-term multinodal load forecasting using a modified general regression neural network. IEEE Trans Power Deliv 2011;26(4):2862-9.

[3] Fan S, Hyndman RJ. Short-term load forecasting based on a semi-parametric additive model. IEEE Trans Power Syst 2012;27(1):134-41.

[4] Taylor JW. Short-term load forecasting with exponentially weighted methods. IEEE Trans Power Syst 2012;27(1):458-64.

[5] Ceperic E, Ceperic V, Baric A. A strategy for short-term load forecasting by support vector regression machines. IEEE Trans Power Syst 2013;28(4):4356-64.

[6] Wang, Senzhang, Jiannong Cao, and Philip Yu. "Deep learning for spatio-temporal data mining: A survey.” IEEE Transactions on Knowledge and Data Engineering (2020).

[7] Yi, Zonggen, and Peter H. Bauer. "Spatiotemporal energy demand models for electric vehicles." IEEE Transactions on Vehicular Technology 65.3 (2015): 1030-1042.

[8] Voulis, Nina, Martijn Warnier, and Frances MT Brazier. "Understanding spatio-temporal electricity demand at different urban scales: A data-driven approach.” Applied Energy 230 (2018): 1157-1171.

[9] Uri, Noel D. "Forecasting peak system load using a combined time series and econometric model.” Applied Energy 4.3 (1978): $219-227$.

[10] Halvorsen, Robert. "Econometric models of US energy demand.” (1978).

[11] Liu, X. Q., B. W. Ang, and T. N. Goh. "Forecasting of electricity consumption: a comparison between an econometric model and a neural network model.” [Proceedings] 1991 IEEE International Joint Conference on Neural Networks. IEEE, 1991.

[12] Chern, Wen S., and Richard E. Just. “Assessing the need for power: a regional econometric model.” Energy Economics 4.4 (1982): $232-239$.

[13] D. Wu, B. Wang, D. Precup and B. Boulet, "Multiple Kernel Learning-Based Transfer Regression for Electric Load Forecasting," in IEEE Transactions on Smart Grid, vol. 11, no. 2, pp. 1183-1192, March 2020, doi: 10.1109/TSG.2019.2933413.

[14] M. Afrasiabi, M. Mohammadi, M. Rastegar, L. Stankovic, S. Afrasiabi and M. Khazaei, "Deep-Based Conditional Probability Density Function Forecasting of Residential Loads," in IEEE Transactions on Smart Grid, vol. 11, no. 4, pp. 3646-3657, July 2020, doi: 10.1109/TSG.2020.2972513.

[15] Z. Cao, C. Wan, Z. Zhang, F. Li and Y. Song, "Hybrid Ensemble Deep Learning for Deterministic and Probabilistic Low-Voltage Load Forecasting," in IEEE Transactions on Power Systems, vol. 35, no. 3, pp. 1881-1897, May 2020, doi: 10.1109/TPWRS.2019.2946701.

[16] Voulis N, Warnier M, Brazier FMT. Impact of service sector loads on renewable resource integration. Appl Energy 2017;205:1311-26.

[17] Mikkola J, Lund PD. Models for generating place and time dependent urban energy demand profiles. Appl Energy 2014;130:256-64.

[18] Keirstead J, Jennings M, Sivakumar A. A review of urban energy system models: approaches, challenges and opportunities. Renew Sustain Energy Rev 2012;16(6):3847-66.

[19] Bourdeau, Mathieu, et al. "Modeling and forecasting building energy consumption: A review of data-driven techniques." Sustainable Cities and Society 48 (2019): 101533.

[20] Newsham, Guy R., and Benjamin J. Birt. "Building-level occupancy data to improve ARIMA-based electricity use forecasts." Proceedings of the 2nd ACM workshop on embedded sensing systems for energy-efficiency in building. 2010.

[21] Yun, Kyungtae, et al. "Building hourly thermal load prediction using an indexed ARX model.” Energy and Buildings 54 (2012): $225-233$.

[22] Dagnely, Pierre, et al. "Predicting hourly energy consumption. Can you beat an autoregressive model." Proceeding of the 24th Annual Machine Learning Conference of Belgium and the Netherlands, Benelearn, Delft, The Netherlands. Vol. 19. 2015.

[23] Amber, Khuram Pervez, et al. "Energy consumption forecasting for university sector buildings.” Energies 10.10 (2017): 1579.

[24] Pulido-Arcas, Jesús A., Alexis Pérez-Fargallo, and Carlos Rubio-Bellido. "Multivariable regression analysis to assess energy consumption and CO2 emissions in the early stages of offices design in Chile.” Energy and Buildings 133 (2016): 738-753.

[25] Egelioglu, Fuat, A. A. Mohamad, and H. Guven. "Economic variables and electricity consumption in Northern Cyprus.” Energy 26.4 (2001): 355-362. 
[26] Mohgram I, Rahman S. Analysis and evaluation of five short-term load fore-casting techniques. IEEE Transactions on Power Systems 1989;4:1484-91.

[27] Papalexopoulos D, Hesterberg TC. A regression based approach to short-term load forecasting. IEEE Transactions on Power Systems 1990;5:1535-50.

[28] Haida T, Muto S. Regression based peak load forecasting using a transforma-tion technique. IEEE Transactions on Power Systems 1994;9:1788-94.

[29] Al-Hamadi, H. M., and S. A. Soliman. "Long-term/mid-term electric load forecasting based on short-term correlation and annual growth." Electric power systems research 74.3 (2005): 353-361.

[30] Chen, Yongbao, et al. "Short-term electrical load forecasting using the Support Vector Regression (SVR) model to calculate the demand response baseline for office buildings.” Applied Energy 195 (2017): 659-670.

[31] Patnaik, Bhaskar, et al. "MODWT-XGBoost based smart energy solution for fault detection and classification in a smart microgrid." Applied Energy 285 (2021): 116457.

[32] Smarra, Francesco, et al. "Data-driven model predictive control using random forests for building energy optimization and climate control." Applied energy 226 (2018): 1252-1272.

[33] Assouline, Dan, Nahid Mohajeri, and Jean-Louis Scartezzini. "Large-scale rooftop solar photovoltaic technical potential estimation using Random Forests.” Applied energy 217 (2018): 189-211.

[34] Aydinalp M, Ismet Ugursal V, Fung AS. Modeling of the appliance, lighting, and space-cooling energy consumptions in the residential sector using neural networks. Applied Energy 2002;71(2):87-110.

[35] Sözen A, Gülseven Z, Arcaklioglu E. Forecasting based on sectoral energy consumption of GHGs in Turkey and mitigation policies. Energy Policy 2007;35(12):6491-505.

[36] Geem ZW, Roper WE. Energy demand estimation of South Korea using artificial neural network. Energy Policy 2009;37(10):4049-54.

[37] González-Romera, E., M. A. Jaramillo-Morán, and D. Carmona-Fernández. "Monthly electric energy demand forecasting with neural networks and Fourier series.” Energy Conversion and Management 49.11 (2008): 3135-3142.

[38] Pao, Hsiao-Tien. “Comparing linear and nonlinear forecasts for Taiwan’s electricity consumption.” Energy 31.12 (2006): $2129-2141$.

[39] Jallal, Mohammed Ali, et al. "A hybrid neuro-fuzzy inference system-based algorithm for time series forecasting applied to energy consumption prediction." Applied Energy 268 (2020): 114977.

[40] Theocharides, Spyros, et al. "Day-ahead photovoltaic power production forecasting methodology based on machine learning and statistical post-processing." Applied Energy 268 (2020): 115023.

[41] Bhattacharyya SC, Timilsina GR. Energy demand models for policy formulation: a comparative study of energy demand models. Policy Res Work Pap 2009;4866.

[42] Swan LG, Ugursal VI. Modeling of end-use energy consumption in the residential sector: a review of modeling techniques. Renew Sustain Energy Rev 2009;13:1819-35. https://doi.org/10.1016/j.rser.2008.09.033.

[43] Mikkola, Jani, and Peter D. Lund. "Models for generating place and time dependent urban energy demand profiles.” Applied Energy 130 (2014): 256-264.

[44] Marquant, Julien F., et al. “A holarchic approach for multi-scale distributed energy system optimisation.” Applied energy 208 (2017): 935953.

[45] Litjens, G. B. M. A., et al. "A spatio-temporal city-scale assessment of residential photovoltaic power integration scenarios.” Solar Energy 174 (2018): 1185-1197.

[46] Eggimann, Sven, Jim W. Hall, and Nick Eyre. "A high-resolution spatio-temporal energy demand simulation to explore the potential of heating demand side management with large-scale heat pump diffusion.” Applied Energy 236 (2019): 997-1010.

[47] Heuberger, Clara F., Praveen K. Bains, and Niall Mac Dowell. “The EV-olution of the power system: A spatio-temporal optimisation model to investigate the impact of electric vehicle deployment.” Applied Energy 257 (2020): 113715.

[48] Yang, Di, et al. "Modeling and spatio-temporal analysis of city-level carbon emissions based on nighttime light satellite imagery." Applied 
Energy 268 (2020): 114696.

[49] Tascikaraoglu, Akin. "Evaluation of spatio-temporal forecasting methods in various smart city applications." Renewable and Sustainable Energy Reviews 82 (2018): 424-435.

[50] Tukey, John W (1977). Exploratory Data Analysis. Addison-Wesley. ISBN 978-0-201-07616-5. OCLC 3058187.

[51] Pang, Yue, et al. "Hierarchical Electricity Time Series Forecasting for Integrating Consumption Patterns Analysis and Aggregation Consistency.” IJCAI. 2018.

[52] W. Kong, Z. Y. Dong, Y. Jia, D. J. Hill, Y. Xu and Y. Zhang, "Short-Term Residential Load Forecasting Based on LSTM Recurrent Neural Network," in IEEE Transactions on Smart Grid, vol. 10, no. 1, pp. 841-851, Jan. 2019, doi: 10.1109/TSG.2017.2753802.

[53] Maaten, Laurens van der, and Geoffrey Hinton. "Visualizing data using t-SNE." Journal of machine learning research 9.Nov (2008): 25792605.

[54] Suganthi, L., and Anand A. Samuel. "Energy models for demand forecasting-A review." Renewable and sustainable energy reviews 16.2 (2012): 1223-1240.

[55] Ioffe S, Szegedy C . Batch normalization: accelerating deep network training by reducing internal covariate shift [C] International Conference on International Conference on Machine Learning. JMLR.org, 2015.

[56] Guo, Diansheng, and Xi Zhu. "Origin-destination flow data smoothing and mapping." IEEE Transactions on Visualization and Computer Graphics 20.12 (2014): 2043-2052.

[57] Wu, Junqi, et al. “ $E^{3}$ : Visual Exploration of Spatiotemporal Energy Demand.” arXiv preprint arXiv:2006.09487 (2020).

[58] Polikar, Robi. "Ensemble based systems in decision making." IEEE Circuits and systems magazine 6.3 (2006): 21-45.

[59] Deb, Chirag, et al. "A review on time series forecasting techniques for building energy consumption." Renewable and Sustainable Energy Reviews 74 (2017): 902-924.

[60] Tsekouras, George J., Nikos D. Hatziargyriou, and Evangelos N. Dialynas. "An optimized adaptive neural network for annual midterm energy forecasting.” IEEE Transactions on Power Systems 21.1 (2006): 385-391.

[61] Marino, Daniel L., Kasun Amarasinghe, and Milos Manic. "Building energy load forecasting using deep neural networks.” IECON 2016-42nd Annual Conference of the IEEE Industrial Electronics Society. IEEE, 2016.

[62] Diederik Kingma and Jimmy Ba. Adam: A method for stochastic optimization. arXiv: 1412.6980, 2014.

[63] Qin Y, Song D, Chen H, et al. A Dual-Stage Attention-Based Recurrent Neural Network for Time Series Forecasting[J]. 2017.

[64] Panapakidis IP, Dagoumas AS. Day-ahead natural gas demand forecasting based on the combination of wavelet transform and ANFIS/genetic algorithm/neural netwrk model. Energy 2017; 118:231-45.

[65] Jokar, Paria, Nasim Arianpoo, and Victor CM Leung. "Electricity theft detection in AMI using customers' consumption patterns." IEEE Transactions on Smart Grid 7.1 (2015): 216-226. 

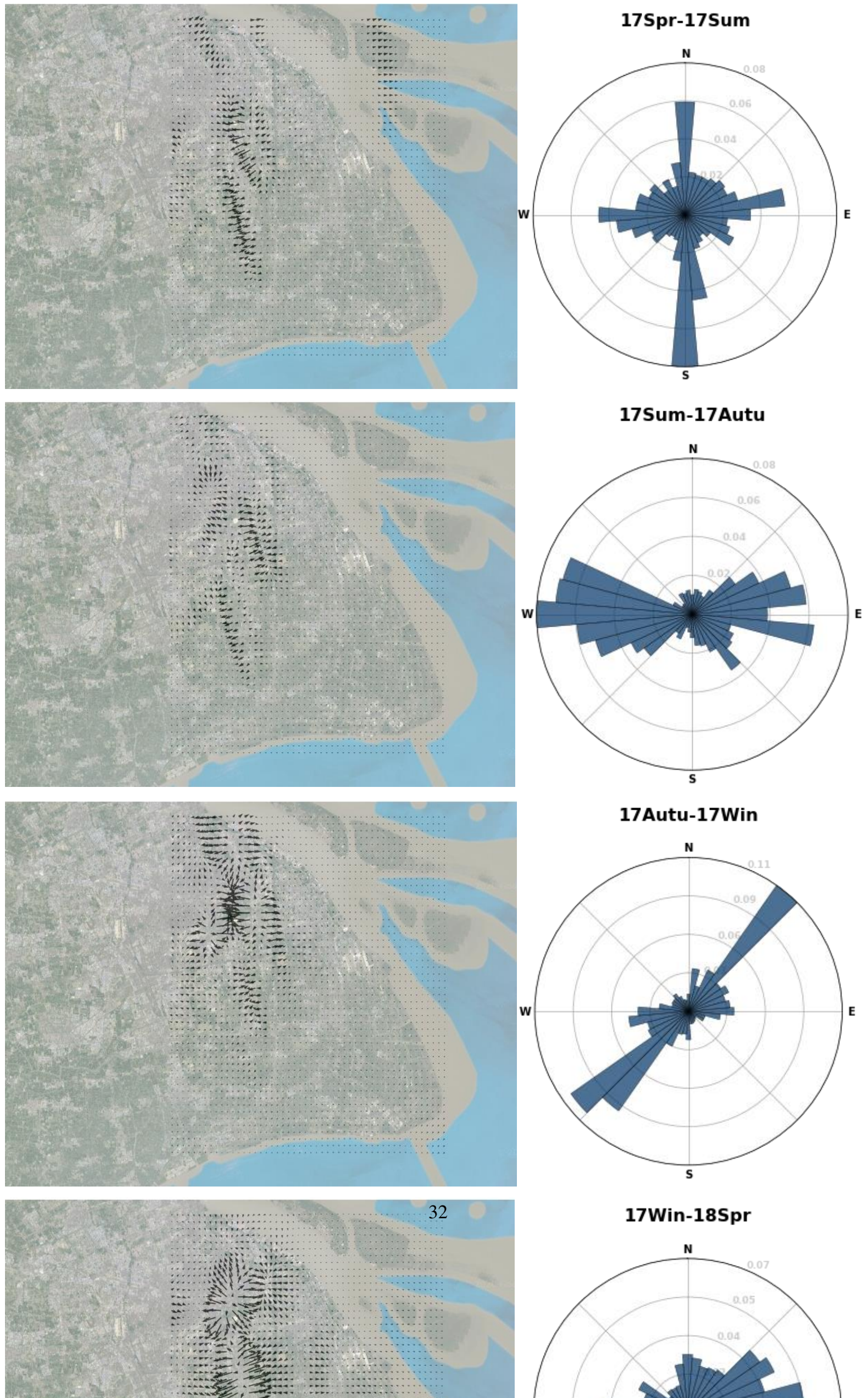


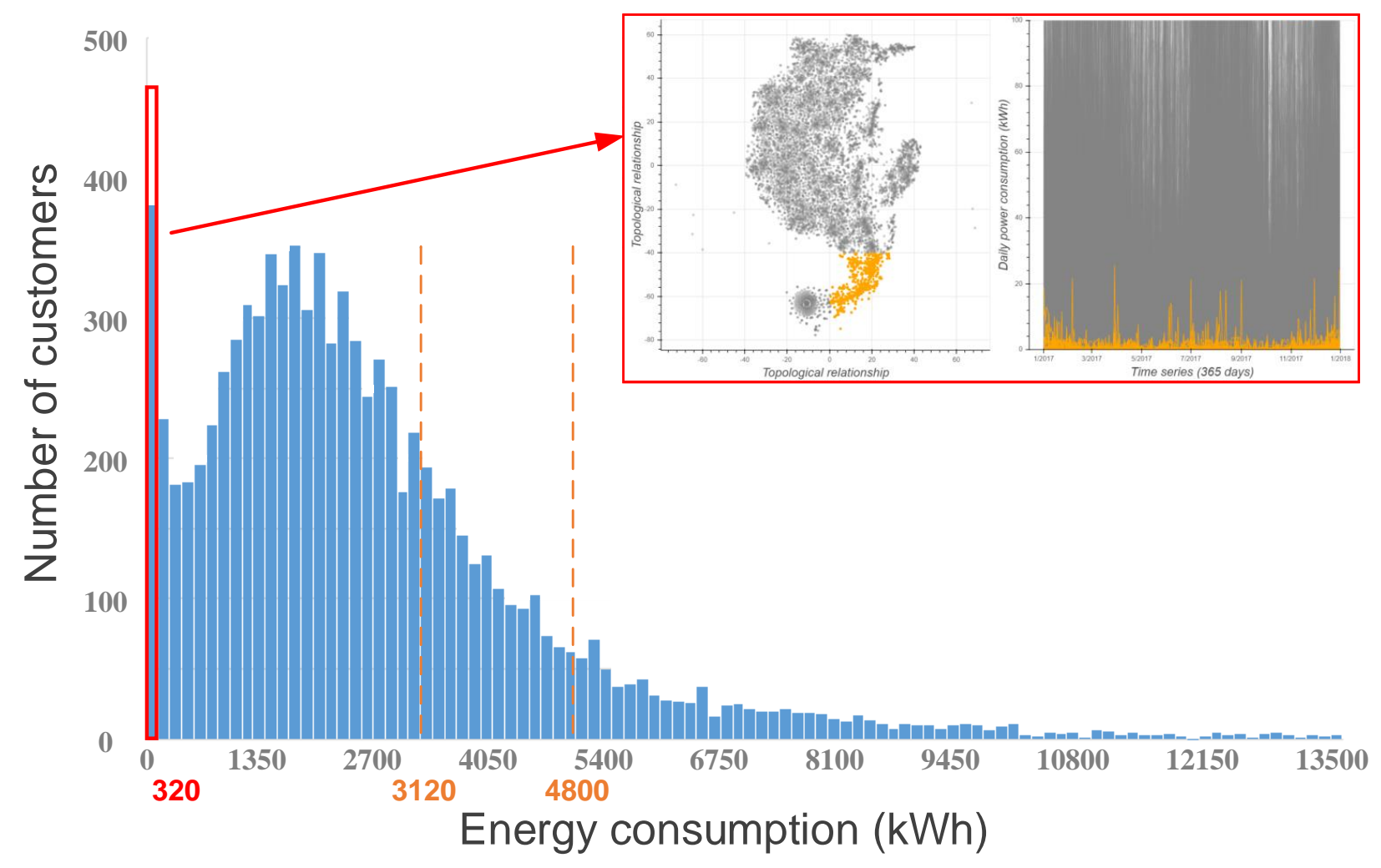

Figure 15: Histogram of household demand distribution. 